\title{
Bleomycin A5 suppresses Drp1-mediated mitochondrial fission and induces apoptosis in human nasal polyp-derived fibroblasts
}

\author{
FAN WU ${ }^{1,2^{*}}$, YUN MA ${ }^{1,2^{*}}$, JINGYI WANG ${ }^{1,2}$, HUASHUANG OU ${ }^{1,2}$, HUA DANG $^{1,2}$, \\ YIQING ZHENG ${ }^{1,2}$, PENG TIAN ${ }^{1,2}$ and HUAZOU ${ }^{1,2}$ \\ ${ }^{1}$ Department of Otorhinolaryngology-Head and Neck Surgery, \\ ${ }^{2}$ Guangdong Provincial Key Laboratory of Malignant Tumor Epigenetics and Gene Regulation, \\ Sun Yat-sen Memorial Hospital, Sun Yat-sen University, Guangzhou, Guangdong 510000, P.R. China
}

Received July 24, 2020; Accepted October 29, 2020

DOI: $10.3892 /$ ijmm.2020.4797

\begin{abstract}
Intralesional injection of bleomycin-A5 (BLE-A5) is a novel treatment for nasal polyps. Our previous study clarified that BLE-A5 could induce nasal polyp-derived fibroblast (NPDF) apoptosis in nasal polyps. However, the detailed mechanisms are still unclear. The present study aimed to determine the effects of BLE-A5 on NPDF mitochondrial dynamics and provide a theoretical basis for the local application of BLE-A5 to treat nasal polyps. In the present study, an in vitro nasal polyp tissue culture model was used to define the BLE-A5 target cell type in nasal polyps. NPDF primary cell culture was used to study the effects of BLE-A5 on the mitochondrial dynamic-related mechanism. The results showed that BLE-A5 treatment of NPDFs caused mitochondrial-mediated apoptosis. Dynamin-related protein 1 (Drp1) was shown to be altered in BLE-A5-treated NPDFs. Drp1 knockdown increased the sensitivity of NPDFs to BLE-A5 and exacerbated mitochondrial dysfunction. BLE-A5 decreased cyclin B1-CDK1 complex-mediated phosphorylation of Drp1 and inhibited Drp1-mediated mitophagy in NPDFs. Overall, the present study concluded that BLE-A5 mainly induces NPDF apoptosis in nasal polyps. BLE-A5 regulates the mitochondria by inhibiting Drp1 activation, resulting in NPDF mitochondrial dynamic disorder and apoptosis.
\end{abstract}

Correspondence to: Dr Hua Zou or Dr Peng Tian, Department of Otorhinolaryngology-Head and Neck Surgery, Sun Yat-sen Memorial Hospital, Sun Yat-sen University, 107 Yan Jiang West Road, Guangzhou, Guangdong 510000, P.R. China

E-mail: zouhua@mail.sysu.edu.cn

E-mail: pengt1205@sina.cn

${ }^{*}$ Contributed equally

Key words: nasal polyps, bleomycin-A5, nasal polyp-derived fibroblasts, dynamin-related protein 1, apoptosis

\section{Introduction}

Nasal polyps (NPs) are characterized by the inflammatory overgrowth of sinonasal tissue (1). Although NPs occur in various diseases, such as malignancy and cystic fibrosis, they are more likely to be associated with chronic rhinosinusitis (CRS), which is also known as chronic rhinosinusitis with nasal polyps (CRSwNP) (2). The prevalence of CRSwNP in European populations is between $2.1 \%$ (France) (3) and $4.4 \%$ (Finland) (4) and is $4.2 \%$ in the United States (5). The prevalence of diagnosis based on endoscopic results in the general population was 2.5 or $2.6 \%$ in Korea (6) and $1.1 \%$ in China (7).

The pathological mechanisms that result in chronic nasal inflammation observed in CRSwNP are not completely understood. Studies have focused on defining the roles of sinonasal epithelial cells, the host immune system and pathogens in CRSwNP pathogenesis (8-10). It was hypothesized that a damaged epithelial barrier could contribute to enhanced exposure to pathogens, antigens and particulates that, in the context of a dysregulated host immune response, may promote chronic inflammation (11). NP tissue is characterized by the predominant infiltration of inflammatory cells, structural fibrosis, stromal tissue edema and tissue remodeling (12). Much of the NP stroma is dense with fibroblasts that produce numerous cytokines, such as transforming growth factor, interleukin 6 and matrix metalloproteinase, thereby causing the infiltration of inflammatory cells $(13,14)$. Moreover, fibroblasts produce extracellular matrix proteins such as collagen I and aggrecan that play critical roles in tissue remodeling (15).

Currently, topical corticosteroids are regarded as the most effective treatment for nasal polyposis (16); however, studies have shown that nasal polyp-derived fibroblasts (NPDFs) are less sensitive to the inhibitory effects of corticosteroids compared with other cell types (17-19). Surgical excision of NPs in patients with significant tissue remodeling may not be beneficial as the NPs can either recur or the surgery can cause severe complications such as adhesion and scar formation $(20,21)$.

Recent studies showed that the treatment of NPs with intralesional bleomycin A5 (BLE-A5) injections is safe and effective, although its underlying mechanism remains unclear (22-24). Studies on the mechanism of BLE in the 
treatment of tumors have shown that BLE can induce cancer cell apoptosis by increasing the levels of reactive oxygen species (ROS) $(25,26)$. In a previous study, we found that BLE could effectively induce NPDF apoptosis via a Bax/Bcl-2-mediated, mitochondria-dependent pathway (27). Moreover, we tested genotoxic and cytotoxic effects in BLE-A5-treated NPDF in our previous researches, including DNA smear testing, cell cytotoxicity assay and cell immigration assay. We found that BLE-A5 can induce DNA fragmentation, reduce cell viability and suppress proliferation in NPDFs $(24,27)$. We also found that NPDF is more sensitive to BLE-A5 administration compared with normal nasal mucosa derived fibroblasts (NMDF) (28). Hence, the present study focused on the detailed mechanism underlying the pro-apoptotic effects of BLE-A5 in NPDFs rather than compare the differences in sensitivity to BLE-A5 treatment in NPDFs and NMDFs.

Mitochondria are highly dynamic organelles that undergo continuous cycles of fusion and fission. Fission is involved in the elimination of damaged mitochondria, which is also known as mitophagy (29). Dynamin-related protein 1 (Drp1) is a highly conserved gene that plays a key role in mitochondrial fission. It was reported that activation of Drp1 protein occurs by phosphorylation of the serine 616 residue [p-Drp1(S616)] and is mediated by the cyclin B1-CDK1 complex, which then causes mitochondrial fission (30-32). Some studies have shown that Drp1 is necessary for eliminating dysfunctional mitochondria via mitophagy (33-35). Moreover, Drp1 is also associated with the translocation of Bax to the mitochondrial outer membrane (36). However, whether BLE can affect Drp1 expression and function in NPDFs is still unclear.

Mitophagy is a catabolic process conserved from yeast to mammals that provides a self-protective mechanism by which cells endure stress. Under stress conditions, dysfunctional mitochondria activate the serine/threonine-protein kinase PINK1 (PINK1)-Parkin-dependent ubiquitination response that involves the remodeling and recycling of mitochondria by mitophagy (37). PINK1 is involved in the degradation of dysfunctional mitochondria, accumulates in depolarized mitochondria and recruits Parkin, which triggers mitochondrial engulfment by the autophagosome (38).

The aim of the present study was to reveal the underlying mechanisms of BLE treatment on NPs and to determine the association between mitophagy and apoptosis in BLE-treated NPDFs.

\section{Materials and methods}

Immunofluorescence analysis of NP fibroblasts and epithelial cell colocalization with TUNEL. In the present study, 12 patients ( 6 females and 6 males; mean age, $42.3 \pm 8.5$ years,) were recruited from the Department of Otorhinolaryngology at Sun Yat-sen Memorial Hospital (Guangzhou, China) between April 2017 to May 2018. All patients were nonsmokers and had either not been treated with glucocorticoids (systemic or topical), antihistamines, non-steroidal anti-inflammatory drugs, or macrolide antibiotics for at least 1 month or who had ceased treatment at least 1 month prior due to lack of alleviation or even exacerbation of symptoms. All participants provided written informed consent in advance, and NP tissues were obtained during surgery. The study was approved by the Ethics Committee of Sun Yat-sen Memorial Hospital (approval no. SYSU81500773).

NP tissue preparation and treatment were performed as described in our previous study (24). After BLE-A5 treatment, a midsagittal section of formalin-fixed (4\% at room temperature for $1 \mathrm{~h}$ ), paraffin-embedded nasal polyp tissue was permeabilized with $0.1 \%$ Triton X-100. Subsequently, the slides were blocked with $10 \%$ goat serum (Sigma-Aldrich; Merck $\mathrm{KGaA}$ ) at room temperature for $1 \mathrm{~h}$, followed by incubation with primary antibodies against vimentin (mouse anti-human; 1:200; cat. no. sc-32322; Santa Cruz Biotechnology, Inc.) and pancytokeratin (CK-pan; rabbit anti-human; cat. no. sc-15367, 1:200; Santa Cruz Biotechnology, Inc.) overnight at $4^{\circ} \mathrm{C}$. The next day, the slides were incubated with Alexa Fluor 594 anti-mouse (goat anti-mouse; 1:200; cat. no. ab150116; Abcam) and Alexa Fluor 647 anti-rabbit secondary antibodies (goat anti-rabbit; 1:200; cat. no. ab150079; Abcam) for $1 \mathrm{~h}$ at room temperature and were washed three times with PBS for $5 \mathrm{~min}$. TUNEL staining was performed to label the 3 '-end of the fragmented DNA in apoptotic cells using a FITC-TUNEL cell apoptosis detection kit (Beyotime Institute of Biotechnology). Slides were mounted on SlowFade mounting media (cat. no. S36937; Thermo Fisher Scientific, Inc.). Cells were observed under a fluorescence microscope at 200 magnification. Green cells were regarded as apoptotic cells, red as fibroblasts and yellow as epithelial cells. Four slides were used to calculate the percentage of apoptotic cells.

Fibroblast isolation and identification. Fibroblasts were isolated from NPs and identified as previously reported (27). Briefly, NP tissues $(\sim 4 \times 4 \mathrm{~mm})$ were cultured in a 6-well dish in DMEM/F-12 (Thermo Fisher Scientific, Inc.) supplemented with $10 \%$ FBS (Gibco; Thermo Fisher Scientific, Inc.), 100 U/ml penicillin and $100 \mathrm{mg} / \mathrm{ml}$ streptomycin in a humidified atmosphere at $37^{\circ} \mathrm{C}$ with $5 \% \mathrm{CO}_{2}$. Fibroblasts were isolated from the tissue via adhesion and migration in a culture dish. When the fibroblast cultures reached $60 \%$ confluence, the remaining tissue was discarded, and adherent cells were digested (in $0.05 \%$ trypsin- $0.02 \%$ EDTA) and seeded in a $150 \mathrm{~cm}^{2}$ dish. Fibroblasts were identified by immunofluorescent staining with CK-pan and vimentin antibodies. Briefly, fibroblasts were cultured on 4-well culture slides, washed with PBS and fixed in 4\% paraformaldehyde at room temperature for $20 \mathrm{~min}$. Subsequently, the cells were permeabilized with $0.1 \%$ Triton X-100 at room temperature for $15 \mathrm{~min}$ and blocked in 5\% non-fat milk-PBS at room temperature for $1 \mathrm{~h}$, followed by incubation with primary antibodies against vimentin (1:200; Santa Cruz Biotechnology, Inc.) or CK-pan (1:200; Santa Cruz Biotechnology, Inc.) overnight at $4^{\circ} \mathrm{C}$. The next day, the slides were incubated with FITC-conjugated secondary antibodies (goat anti-mouse; 1:200; cat. no. ab6785; Abcam and goat anti-rabbit; 1:200; cat. no. ab6717; Abcam) at room temperature for $30 \mathrm{~min}$ and washed three times with PBS for $5 \mathrm{~min}$. The cells were incubated with DAPI $(1: 1,000)$ at room temperature for $5 \mathrm{~min}$ to label the nuclei, and the stained slides were examined under a fluorescence microscope (Olympus Corporation) at x40 magnification.

Cell treatments. To examine the effect of BLE dose and exposure time on the expression of mitochondrial apoptotic pathway-associated proteins in NPDFs, cells were treated with 
various concentrations of BLE-A5 $(0,50,100,200$ or $400 \mu \mathrm{M}$; cat. no. 19692; Cayman Chemical Company) for $48 \mathrm{~h}$ or with $200 \mu \mathrm{M}$ BLE-A5 for various durations $(0,6,12,24,48$ or $72 \mathrm{~h}$ ). In a previous study, we found that treatment of NPDFs with $200 \mu \mathrm{M}$ BLE-A5 for $48 \mathrm{~h}$ could effectively induce NPDF apoptosis, and hence this treatment protocol (200 $\mu$ M BLE-A5 for $48 \mathrm{~h}$ ) was used for most of the experiments in the present study. Aliquoted BLE was dissolved in PBS at a concentration of $20 \mathrm{mM}$ and stored at $-30^{\circ} \mathrm{C}$ before use. Validated small interfering RNA (siRNA) that specifically targeted Drp1 (50 nM; target sequence, 5'-CAAGGAGCCAGTCAA ATTA-3'; Shanghai GenePharma Co., Ltd.) was used to knock down Drp1. Cells were transfected using Lipofectamine ${ }^{\circledR} 3000$ (Invitrogen; Thermo Fisher Scientific, Inc.) according to the manufacturer's instructions. The control groups consisted of untransfected NPDFs and NPDFs transfected with scrambled siRNA (50 nM; Si-Ctrl; 5'-UUCUCCGAACGUGUCACGU-3'; Shanghai GenePharma Co., Ltd.). Henceforth, Si-Drp1 refers to siRNA targeting Drp1, Si-Ctrl refers to nonspecific siRNA and untransfected cells are referred to as the negative control (NC). Both si-Drp1 and si-Ctrl were transfected for $72 \mathrm{~h}$ before subsequent treatment. Knockdown efficiency was determined by PCR and western blotting. To show the effects of the Cyclin B1-CDK1 complex on p-Drp1 levels, NPDFs were pretreated with the CDK1 inhibitor RO-3306 (20 $\mu \mathrm{M}$; cat. no. 872573-93-8; Sigma-Aldrich; Merck KGaA) for $24 \mathrm{~h}$ before p-Drpl(S616) (1:1,000; cat. no. 3455; Cell Signaling Technology, Inc.) levels were analyzed by western blotting. RO-3306 was diluted in DMSO; thus, the control group was treated with an equal dose of DMSO (vehicle).

Reverse transcription-PCR (RT-PCR). Untransfected (NC), Si-Ctrl-transfected, or Si-Drp1-transfected NPDFs were homogenized in $1 \mathrm{ml}$ TRIzol ${ }^{\circledR}$ reagent (Invitrogen; Thermo Fisher Scientific, Inc.). Total RNA was extracted, and $2 \mu \mathrm{g}$ of RNA/sample was reverse transcribed into cDNA using a reverse transcription kit according to the manufacturer's protocol (cat. no. FSQ-101; Toyobo Life Science). Subsequently, using the Roche PCR system (Roche Diagnostics), PCR was performed on a reaction mixture containing $2 \mu \mathrm{l}$ cDNA, $0.2 \mu 1$ of each primer (forward and reverse), $5 \mu 12 \mathrm{X} \mathrm{PCR}$ master mix reaction buffer (cat. no. F1696K; Toyobo Life Science) and deionized water to a final volume of $10 \mu \mathrm{l}$. The following thermocycling conditions were used for the PCR: Initial denaturation at $90^{\circ} \mathrm{C}$ for $30 \mathrm{sec}$, followed by 40 cycles of $94^{\circ} \mathrm{C}$ for $30 \mathrm{sec}, 60^{\circ} \mathrm{C}$ for $30 \mathrm{sec}$ and $72^{\circ} \mathrm{C}$ for $1 \mathrm{~min}$. Equal volumes of PCR products were evaluated by $5 \%$ agarose gel electrophoresis and visualized using the Bio-Rad Gel Doc XR documentation system (Bio-Rad Laboratories, Inc.). DNA bands were measured using ImageJ (version 1.6; National Institutes of Health) The primer sequences used for PCR are listed as follows: Drp1 forward, 5'-ATATGCCGAGTTCCT GCACTG-3' and reverse, 5'-AGTAGACGCGGAGGTTGA TAG-3' (39) and GAPDH forward, 5'-CAGTGCCAGCCT CTGCTCAT-3' and reverse, 5'-ATACTCAGCACCAGCAC AT-3' (40).

Flow cytometry with Annexin V and propidium iodide (PI). An Annexin V/PI apoptosis kit (Thermo Fisher Scientific, Inc.) was used to assess BLE-induced NPDF apoptosis according to the manufacturer's instructions. Briefly, Si-Ctrlor Si-Drp1-transfected NPDFs were incubated in 6-well plates at a density of $5 \times 10^{6}$ cells/well with DMEM/F12 supplemented with $10 \%$ FBS and various concentrations of BLE $(0,50$, or $200 \mu \mathrm{M})$ for 24 or $48 \mathrm{~h}$. The cultured fibroblasts were gently suspended in binding buffer and incubated in the dark at room temperature for $15 \mathrm{~min}$ with $5 \mu \mathrm{l}$ Annexin V-FITC and $10 \mu \mathrm{l}$ PI. Annexin V-FITC- and PI-labeled cells were analyzed using a flow cytometer (BD Biosciences). Dot plots (with PI on the $y$-axis and Annexin V-FITC on the x-axis) were used to identify viable cells, which appear negative for PI and Annexin V-FITC staining. Cells in the early stages of apoptosis appear as Annexin V-positive and PI-negative, while cells in late apoptosis or full necrosis show both Annexin V-FITC-positive and PI-positive staining.

Mitochondrial and cytosolic fractionation. Cells were lysed with Buffer A [0.25 M sucrose, $10 \mathrm{mM}$ Tris- $\mathrm{HCl}(\mathrm{pH} 7.5)$, $10 \mathrm{mM} \mathrm{KCl}, 1.5 \mathrm{mM} \mathrm{MgCl}_{2}, 1 \mathrm{mM}$ EDTA, $1 \mathrm{mM}$ dithiothreitol, and $0.1 \mathrm{mM}$ PMSF] with a homogenizer. The homogenate was centrifuged at $750 \mathrm{xg}$ for $10 \mathrm{~min}$ at $4^{\circ} \mathrm{C}$, and the supernatant was collected and centrifuged at $10,000 \mathrm{xg}$ for $30 \mathrm{~min}$ at $4^{\circ} \mathrm{C}$. After the second centrifugation, the supernatant was collected as the cytosolic fraction, and the pellet was collected as the mitochondrial fraction. The pellets were resuspended in Buffer B [0.25 M sucrose, $10 \mathrm{mM}$ Tris- $\mathrm{HCl}$ (pH 7.5), $10 \mathrm{mM}$ $\mathrm{KCl}, 1.5 \mathrm{mM} \mathrm{MgCl}{ }_{2}, 1 \mathrm{mM}$ EDTA, $1 \mathrm{mM}$ dithiothreitol, $0.1 \mathrm{mM}$ PMSF and $1 \% \mathrm{NP} 40]$.

Western blotting. Cells from different groups were homogenized in RIPA buffer (Sigma Aldrich; Merck KGaA) containing $1 \mathrm{mM}$ PMSF, protease inhibitor cocktail and phosphatase inhibitor cocktail (Roche Diagnostics) on ice for $30 \mathrm{~min}$. Protein concentration was determined using a BCA protein assay kit (Beyotime Institute of Biotechnology). Equal amounts of protein ( $10 \mu \mathrm{g} / \mathrm{lane})$ were separated using 10\% SDS-PAGE and transferred onto PVDF membranes (EMD Millipore). The membranes were blocked in 5\% non-fat milk in TBS for $1 \mathrm{~h}$ at room temperature. Subsequently, the membranes were incubated overnight at $4^{\circ} \mathrm{C}$ with primary antibodies against Drp1 (1:500), p-Drp1 (S616) (1:500; cat. no. 3455), Bax (1:1,000; cat. no. 5023), Bcl-2 (1:1,000; cat. no. 15071), cleaved caspase-9 (1:1,000; cat. no. 20750), PINK1 (1:1,000; cat no. 6946), Parkin (1:1,000; cat. no. 4211), LC3B (1:1,000; cat. no. 3868), cyclooxygenase (COX) IV $(1: 1,000 ;$ cat. no. 4850$), \alpha$-tubulin $(1: 1,000 ;$ cat. no. 2125$), \beta$-actin (1:3,000; cat. no. 4970) or GAPDH (1:10,000; cat. no. 5174). After washing TBS with $5 \%$ Tween-20, the membranes were incubated with horseradish peroxidase-conjugated anti-rabbit $(1: 2,000$; cat. no. 7074) or anti-mouse $(1: 2,000$; cat. no. 7076) secondary antibodies for $1 \mathrm{~h}$ at room temperature and then visualized using an enhanced chemiluminescence kit (EMD Millipore). All aforementioned antibodies were purchased from Cell Signaling Technology, Inc. Band images were captured using the Bio-Rad Gel Doc XR documentation system (Bio-Rad Laboratories, Inc.). Relative protein expression levels were determined by ImageJ (version 1.6; National Institutes of Health) and standardized to GAPDH or $\beta$-actin. The protein levels of $\alpha$-tubulin were used to confirm equal loading of cytosolic proteins, and the protein levels of COX IV were used to confirm equal loading of mitochondrial proteins. 
Mitochondrial morphology analysis. To examine the effects of Drp1 on the mitochondrial morphology of BLE-treated NPDFs, Si-Ctrl- or Si-Drp1-transfected cells were cultured in 24-well culture plates at a density of $1 \times 10^{5}$ cells/well and treated with $200 \mu \mathrm{M}$ BLE-A5 for $48 \mathrm{~h}$. Subsequently, the cells were incubated with $500 \mathrm{nM}$ MitoTracker Red (MitoRed; Thermo Fisher Scientific, Inc.) mitochondrial dye in a humidity-controlled environmental chamber at $37^{\circ} \mathrm{C}$ with $5 \% \mathrm{CO}_{2}$ for $30 \mathrm{~min}$. After fixation in $4 \%$ paraformaldehyde at room temperature for $20 \mathrm{~min}$, cells were permeabilized with $0.1 \%$ Triton X-100 at room temperature for $15 \mathrm{~min}$ and blocked in 5\% non-fat milk-PBS at room temperature for $1 \mathrm{~h}$. The cells were then incubated with primary antibodies against p-Drp1 $(1: 200)$ or LC3B $(1: 200)$ overnight at $4^{\circ} \mathrm{C}$. The next day, the slides were incubated with FITC-conjugated secondary antibodies (1:200; cat. no. ab6717; Abcam) for 30 min and washed three times with PBS for $5 \mathrm{~min}$. The cells were incubated with DAPI $(1: 1,000)$ at room temperature for $5 \mathrm{~min}$ to label the nuclei. The stained slides were examined under a fluorescence microscope (Olympus Corporation) at x40 magnification.

Superoxide analysis. Cells were stained with MitoSOX Red (Thermo Fisher Scientific, Inc.), and ROS production was measured by flow cytometry. Si-Ctrl- or Si-Drp1-transfected NPDFs were treated with or without $200 \mu \mathrm{M}$ BLE-A5 for $48 \mathrm{~h}$ and then incubated with $5 \mu \mathrm{M}$ MitoSOX Red for $30 \mathrm{~min}$. The cells were collected and washed with PBS, and fluorescence was analyzed using FACSAria III (BD Biosciences).

Mitochondrial DNA (mtDNA) analysis. After BLE-A5 treatment, total cellular DNA of Si-Ctrl- or Si-Drp1-transfected NPDFs was extracted using DNAzol ${ }^{\circledR}$ reagent (Invitrogen; Thermo Fisher Scientific, Inc.). The relative mtDNA copy number was defined as the ratio of mtDNA [represented by the NADH-ubiquinone oxidoreductase chain 1 (ND1) gene] to nuclear DNA (nDNA, represented by the $\beta$-actin gene), as assessed by quantitative PCR (qPCR). SYBR green was used as a fluorophore (Toyobo Life Science). The following thermocycling conditions were used for the qPCR: Initial denaturation at $95^{\circ} \mathrm{C}$ for $5 \mathrm{~min}$; followed by 45 cycles of $95^{\circ} \mathrm{C}$ for $10 \mathrm{sec}, 60^{\circ} \mathrm{C}$ for $10 \mathrm{sec}$ and $72^{\circ} \mathrm{C}$ for $20 \mathrm{sec}$. Finally, a melting curve was plotted to confirm the presence of a single PCR product at the following conditions: $95^{\circ} \mathrm{C}$ for $5 \mathrm{sec}, 66^{\circ} \mathrm{C}$ for $1 \mathrm{~min}$ and gradual increase in temperature up to $97^{\circ} \mathrm{C}$ for fluorescence acquisition. Analysis of the mtDNA/nDNA ratio was calculated using the $2^{-\Delta \Delta \mathrm{Cq}}$ method (41). The following primer pairs were used for the qPCR: ND1 forward, 5'-CCC TAAAACCCGCCACATCT-3' and reverse, 5'-GAGCGATGG TGAGAGCTAAGGT-3' and $\beta$-actin forward, 5'-ATGAAG ATCAAGATCATTGCTCCTC-3' and reverse, 5'-ACATCT GCTGGAAGGTGGACA-3'. Two independent PCRs were performed for mitochondrial and nuclear DNA in each sample.

Mitochondrial membrane potential analysis. The mitochondrial membrane potential was analyzed using the radiometric cationic fluorescent dye $\mathrm{JC}-1$. The cells were stained with $10 \mu \mathrm{M} \mathrm{JC}-1$ at $37^{\circ} \mathrm{C}$ for $10 \mathrm{~min}$. The change in fluorescence intensity of the dye was measured in the red and green channels by flow cytometry and normalized to that of the control group.
ATP analysis. ATP was measured by a bioluminescence assay using an ATP determination kit (Molecular Probes; Thermo Fisher Scientific, Inc.). Briefly, Si-Ctrl- or Si-Drp1-transfected NPDFs were treated with $200 \mu \mathrm{M}$ BLE-A5 for $48 \mathrm{~h}$ and resuspended in reaction buffer containing $1 \mathrm{mM}$ dithiothreitol, $0.5 \mathrm{mM}$ D-luciferin and $12.5 \mu \mathrm{g} / \mathrm{ml}$ firefly luciferase. After 15 min of incubation, cellular ATP was measured using a microplate luminometer (Molecular Devices, LLC).

Co-immunoprecipitation. In brief, NPDFs were treated without or with $200 \mu \mathrm{M}$ BLE-A5 for $48 \mathrm{~h}$, collected, washed with ice-cold PBS, and lysed with lysis buffer containing $50 \mathrm{mmol} / 1$ Tris (pH 8.0), $150 \mathrm{mmol} / 1 \mathrm{NaCl}$ and 1\% NP40. Lysates containing $300 \mu \mathrm{g}$ protein was incubated overnight at $4^{\circ} \mathrm{C}$ with $10 \mu \mathrm{g}$ anti-cyclin B1 antibody (cat. no. ab32053; Abcam). Protein A/G PLUS-agarose (50 $\mu \mathrm{l}$; Santa Cruz Biotechnology, Inc.) was subsequently added to each sample, and the incubation was continued for an additional $3 \mathrm{~h}$ at $4{ }^{\circ} \mathrm{C}$ with gentle shaking. The immunoprecipitates were subjected to SDS-PAGE followed by immunoblotting using anti-CDK1 $(1: 1,000)$ or anti-cyclin B1 (1:1,000; Santa Cruz Biotechnology, Inc.) antibodies. Electrophoresis, western blotting and imaging procedures were performed as described in under the Western blotting subsection.

Statistical analysis. All experiments were repeated three times or more to provide sufficient data for statistical analysis. The differences were analyzed with Student's t-test or one-way ANOVA followed by Dunnett's test or Sidak's multiple comparisons test. Statistical tests were conducted using GraphPad Prism version 5 (GraphPad Software, Inc.). Statistical significance was determined at the $95 \%$ confidence level. $\mathrm{P}<0.05$ was considered to indicate a statistically significant difference.

\section{Results}

BLE-A5 mainly induces fibroblast apoptosis in nasal polyps. As shown in Fig. 1A and B, immunofluorescence analysis of TUNEL co-localization showed that BLE-A5 induced apoptosis (green) mainly in fibroblasts (red, 66 $\pm 12 \%$ ) but not epithelial cells (yellow, $21 \pm 10 \%$ ). Based on these results, the present study focused on the mechanism by which BLE-A5 triggered NPDF apoptosis in subsequent experiments.

Identification of NPDFs. Isolated fibroblasts showed vimentin-positive and CK-pan-negative immunofluorescence staining, with a spindle- and nest-like distribution. These features were consistent with fibroblast characteristics (Fig. 1C-E), which showed the positive identification of NPDFs.

BLE-A5 treatment induces apoptosis in human NPDFs and alters Drpl expression. In a previous study (25), we found that BLE-A5 could induce NPDF apoptosis in a time- and dose-dependent manner. The present study confirmed the pro-apoptotic effects of BLE-A5 by western blot analysis of Bax, Bcl-2 and cleaved caspase-9. BLE-A5 increased the levels of cleaved caspase- 9 and decreased the levels of Bcl-2 (Fig. 2), suggesting the possible involvement of a mitochondria-mediated apoptosis pathway. Even a low dose of 

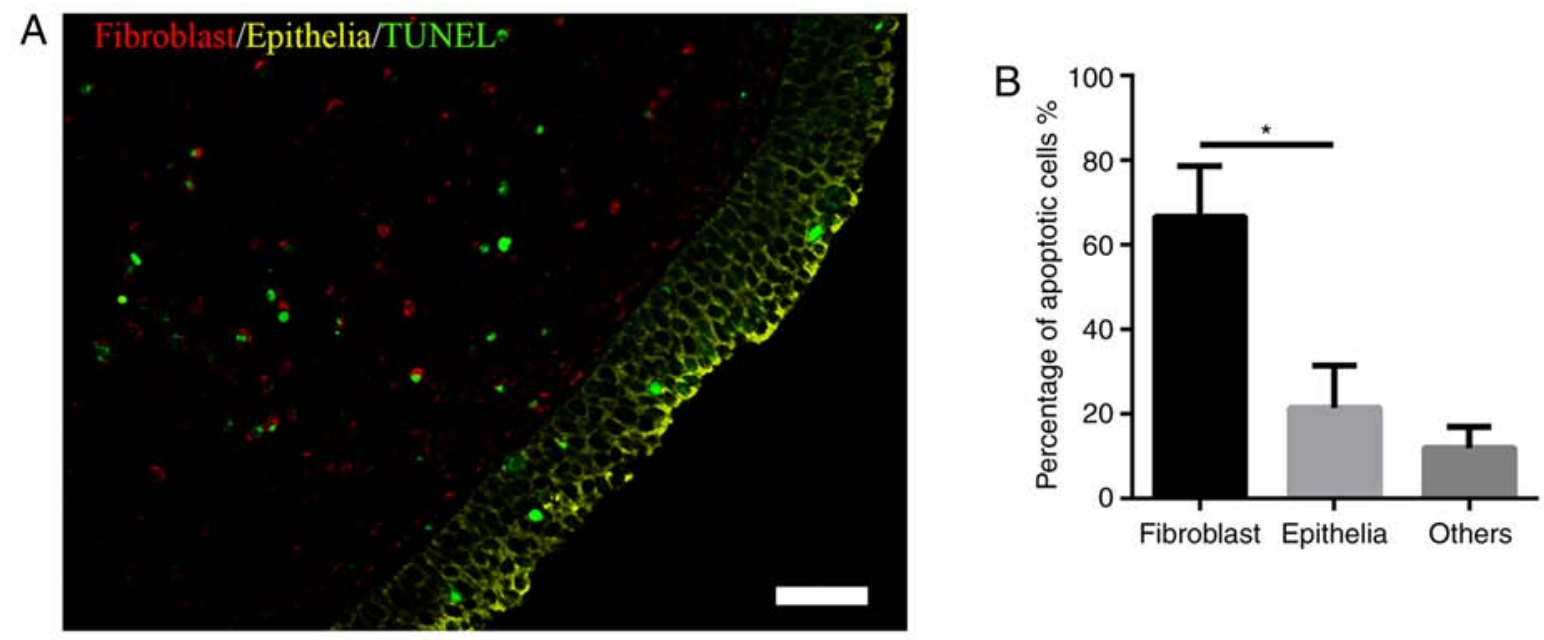

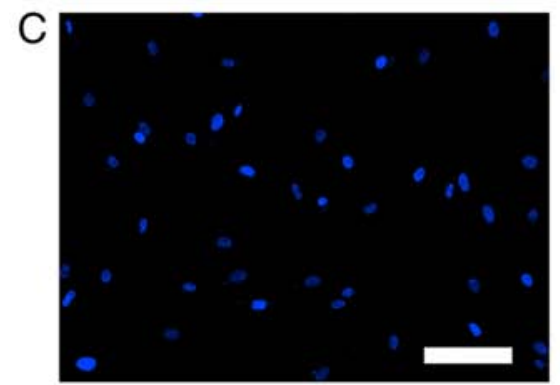

Control

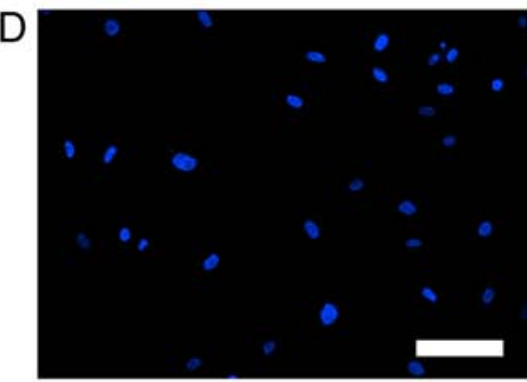

CK-pan

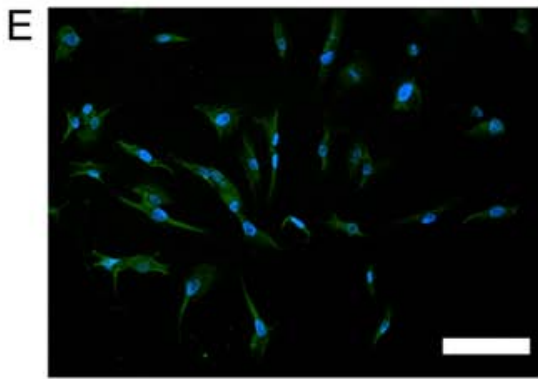

Vimentin

Figure 1. Immunofluorescence and TUNEL colocalization to identify BLE-A5-induced apoptotic cells in nasal polyps and the identification of isolated NPDFs. (A) BLE-A5-treated nasal polyp slides stained with the apoptosis marker TUNEL (green), fibroblast marker (red) and epithelial marker (yellow). Scale bar, $100 \mu \mathrm{m}$. (B) Statistical analysis of TUNEL-positive cells in different groups. $\mathrm{n}=4$. ${ }^{*} \mathrm{P}<0.05$. Immunofluorescence staining to identify isolated NPDFs using (C) PBS as a control group, (D) CK-pan as a negative marker (E) and vimentin as a positive marker. Scale bar, $100 \mu$ m. Data were analyzed by one-way ANOVA followed by Sidak's multiple comparisons test. NPDF, nasal polyp-derived fibroblast; BLE-A5, bleomycin A5; CK-pan, pancytokeratin.

BLE-A5 $(25 \mu \mathrm{M})$ altered the Bax/Bcl-2 ratio and the expression of cleaved caspase-9 (Fig. 1D and F). Compared to the control group, the ratio of Bax/Bcl-2 and cleaved caspase- 9 expression increased significantly after 12 and $48 \mathrm{~h}$ BLE-A5 treatment, respectively. These results are consistent with our previous findings that exposure to $200 \mu \mathrm{M}$ BLE-A5 for $48 \mathrm{~h}$ can effectively induce apoptosis in NPDFs.

To examine the effects of BLE-A5 on mitochondrial fusion, the protein levels of Drp1 in NPDFs were measured by western blotting. As shown in Fig. 2A, BLE-A5 treatment for $48 \mathrm{~h}$ showed a dose-dependent decrease in Drp1 protein in NPDFs. When testing the effect of the duration of BLE exposure on NPDFs, it was found that Drp1 expression increased at the 12-24 h time points and then sharply decreased after $48 \mathrm{~h}$.

Drpl knockdown increases the sensitivity of NPDFs to BLE. To study the functional role of Drpl in BLE-treated NPDFs, NPDFs were transiently transfected with siRNA to downregulate the expression of Drp1 (Si-Drp1) or with nonspecific siRNA (Si-Ctrl) as a control. Untransfected NPDFs were also used as NCs. Transfection of cells with Si-Drp1 significantly decreased Drp1 mRNA (Fig. 3A) and protein expression (Fig. 3B).

Following siRNA transfection, NPDFs were exposed to 50 or $200 \mu \mathrm{M}$ BLE-A5 for $48 \mathrm{~h}$. The levels of apoptosis were then assessed using an Annexin V/PI apoptosis kit (Fig. 3C). The results showed that the percentage of apoptotic NPDFs was significantly increased after Drp1 knockdown and exposure to both 50 and $200 \mu \mathrm{M}$ BLE-A5 (Fig. 3D).

Next, the present study analyzed proteins associated with the mitochondria-mediated apoptotic pathway by western blotting (Fig. 3E). As shown in Fig. 3F, the expression levels of Drp1 in NPDFs after siRNA transfection was similar to that of NPDFs treated with $200 \mu \mathrm{M}$ BLE for $48 \mathrm{~h}$. Additionally, Si-Drp1-transfected NPDFs that were treated with the same dose of BLE showed a significant decrease in Drp1 expression (Fig. 3F). The Bax/Bcl-2 ratio and cleaved caspase-9 expression were increased by BLE-A 5 treatment but did not change in the Si-Drp1-transfected group compared to the control group. Furthermore, Si-Drp1-transfected NPDFs exposed to BLE-A5 exhibited significantly increased $\mathrm{Bax} / \mathrm{Bcl}-2$ ratios and cleaved caspase- 9 expression compared with the $\mathrm{Si}-\mathrm{Ctrl}$ group treated with the same dose of BLE (Fig. 3G and H).

BLE-A5 inhibits Drp1-mediated mitochondrial fission in $N P D F s$. Fluorescence microscopy was performed to directly visualize the changes in mitochondrial morphology in NPDFs. MitoRed was used to identify mitochondria, and a Drp1 primary antibody linked to a FITC-tagged secondary antibody was used to detect Drp1. As shown in Fig. 4, the $\mathrm{Si}-\mathrm{Ctrl}$ group showed robust green fluorescence in the cytoplasm, which co-localized with MitoRed fluorescence in 
A

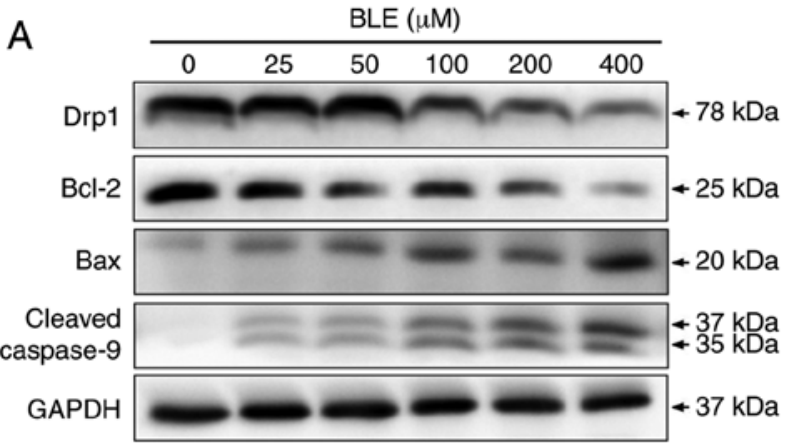

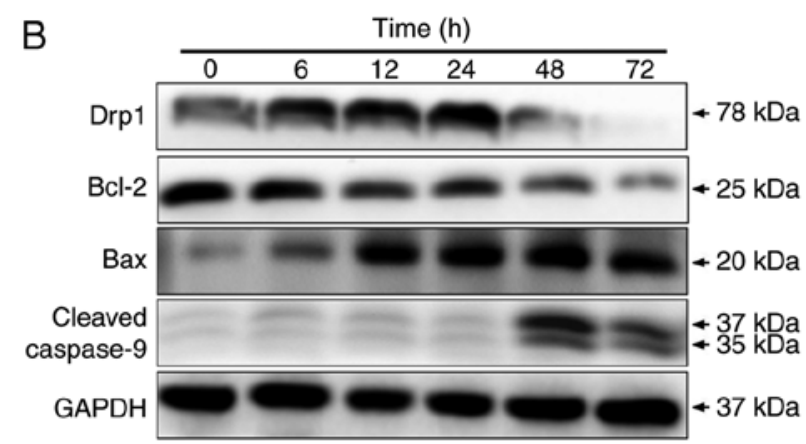
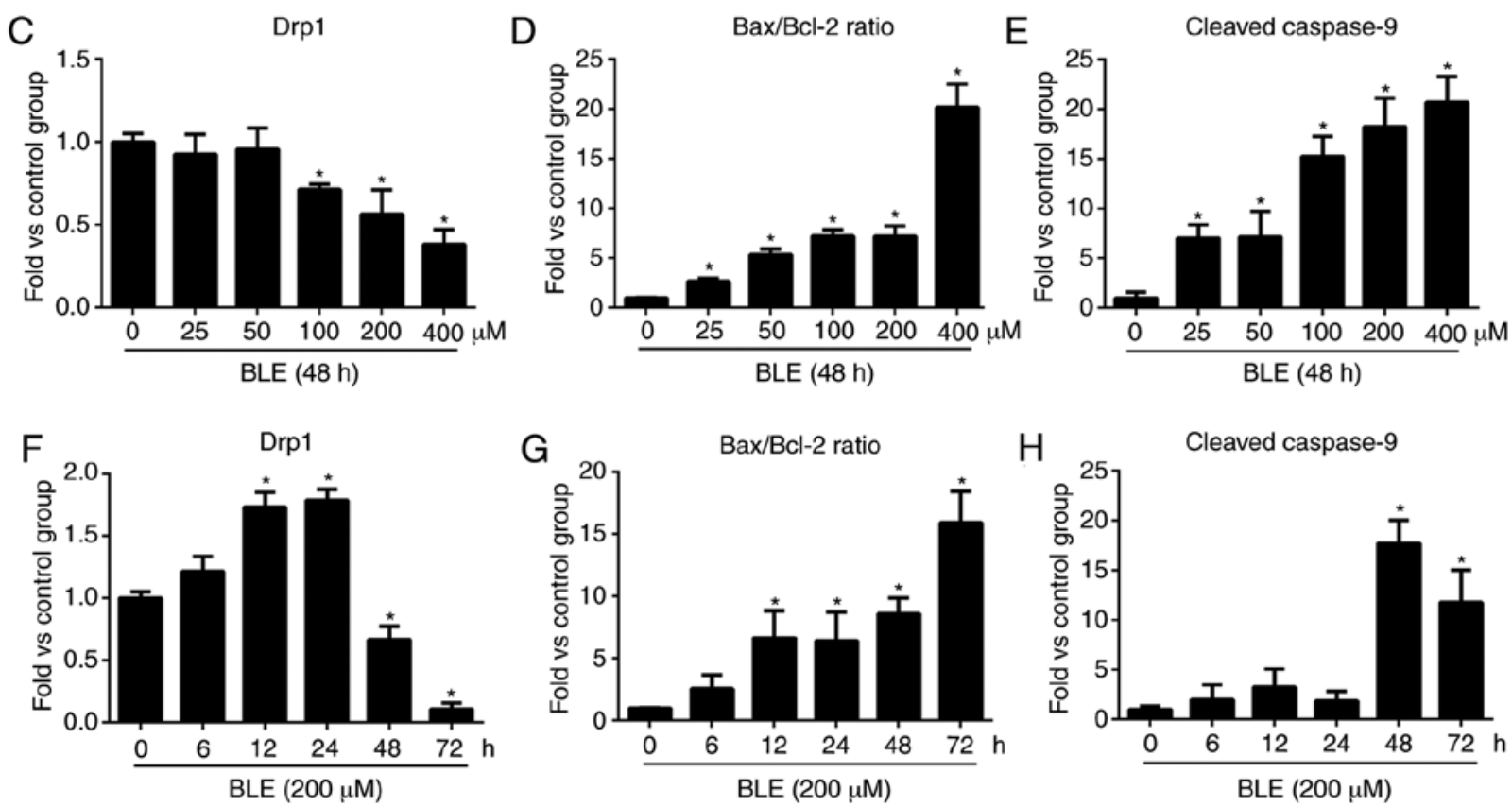

Figure 2. Western blot analysis of the effects of different exposure times and doses of BLE-A5 on Drp1 and mitochondria-mediated apoptotic protein expression. Treatment of nasal polyp-derived fibroblasts with (A) a concentration gradient of BLE-A5 for 48 h and (B) a time course with $200 \mu \mathrm{M}$ BLE-A5 treatment. Quantification of the results in (A) showing the fold-change in (C) Drp1 expression, (D) Bax/Bcl-2 ratio and (E) cleaved caspase-9 expression normalized to the internal reference, GAPDH. Quantification of the results in (B) showing the fold-change in (F) Drp1 expression, (G) Bax/Bcl-2 ratio and $(\mathrm{H})$ cleaved caspase- 9 expression normalized to the internal reference, GAPDH. Values are presented as the mean \pm standard deviation. $n=3$. ${ }^{*} \mathrm{P}<0.05$ vs. controls. Data were analyzed by one-way ANOVA and Dunnett's post hoc test for multiple comparisons. C-Cas9, cleaved caspase-9; BLE-A5, bleomycin A5; Drp1, dynamin-related protein 1.

the form of short tubules or round fragments. By contrast, Si-Drp1 transfection markedly decreased the green (Drp1) fluorescence, and the mitochondrial morphology was altered to long, interconnected tubular networks. Similar phenotypes were observed in the Si-Ctrl group treated with BLE-A5. However, when Si-Drp1-transfected NPDFs were treated with BLE-A5, Drp1 fluorescence was extremely weak and the mitochondria appeared as long tubular networks. These results indicated that BLE treatment changed the mitochondrial morphology by decreasing Drp1 expression.

Drpl knockdown aggravates BLE-induced mitochondrial dysfunction in NPDFs. To determine the effects of Drp1 on BLE-induced mitochondrial dysfunction, the present study measured exogenous ROS levels, mtDNA levels, ATP levels and mitochondrial membrane potential $(\Delta \psi \mathrm{m})$ in Si-Ctrlor Si-Drp1-transfected NPDFs treated with or without BLE.
As shown in Fig. 5A and B, the levels of ROS in Si-Ctrl- and Si-Drp1-transfected NPDFs were enhanced by BLE-A5 treatment, but Drp1 knockdown alone did not change ROS levels compared with controls. Si-Drp1-transfected NPDFs showed higher levels of superoxide accumulation compared with Si-Ctrl-transfected cells when exposed to the same dose of BLE-A5 (200 $\mu \mathrm{M}$ for $48 \mathrm{~h}$ ). Similar results were observed for mtDNA levels; mtDNA was increased by BLE-A5 exposure in Si-Ctrl- and Si-Drp1-transfected NPDFs but showed no significant change in response to Drp1 knockdown alone. Si-Drp1-transfected NPDFs were more likely to undergo mtDNA duplication compared with the Si-Ctrl group when treated with BLE-A5 (Fig. 5C). These changes were consistent with the decrease in mitochondrial ATP production (Fig. 5D). Si-Drp1 NPDFs generated less ATP compared with the Si-Ctrl group when exposed to BLE-A5, indicating increased impairments in the oxidative phosphorylation ability of mitochondria. When JC-1 was used to measure the $\Delta \psi \mathrm{m}$ levels (Fig. $5 \mathrm{E}$ and $\mathrm{F}$ ), it was found that the mitochondrial potential of Si-Drp1-transfected NPDFs 
A

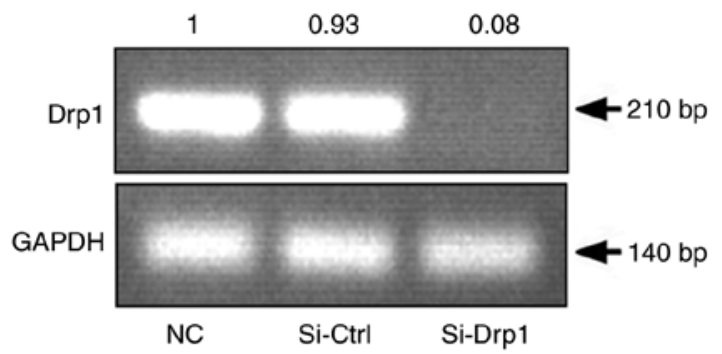

B

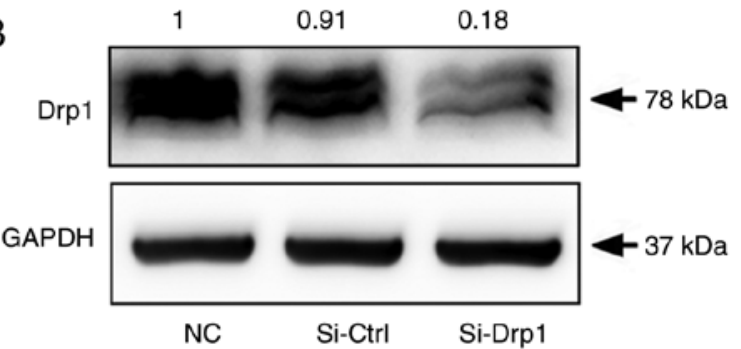

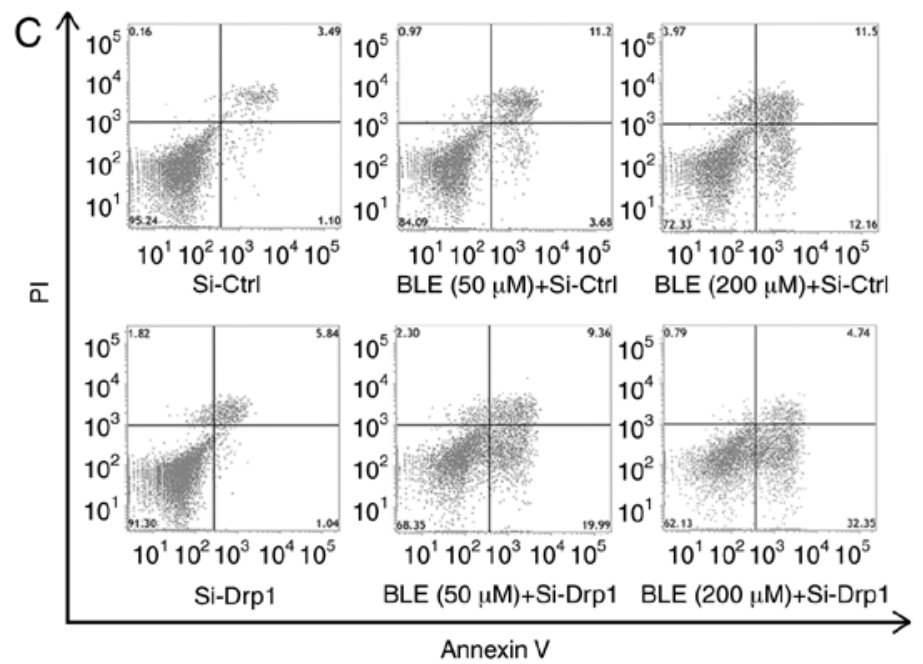
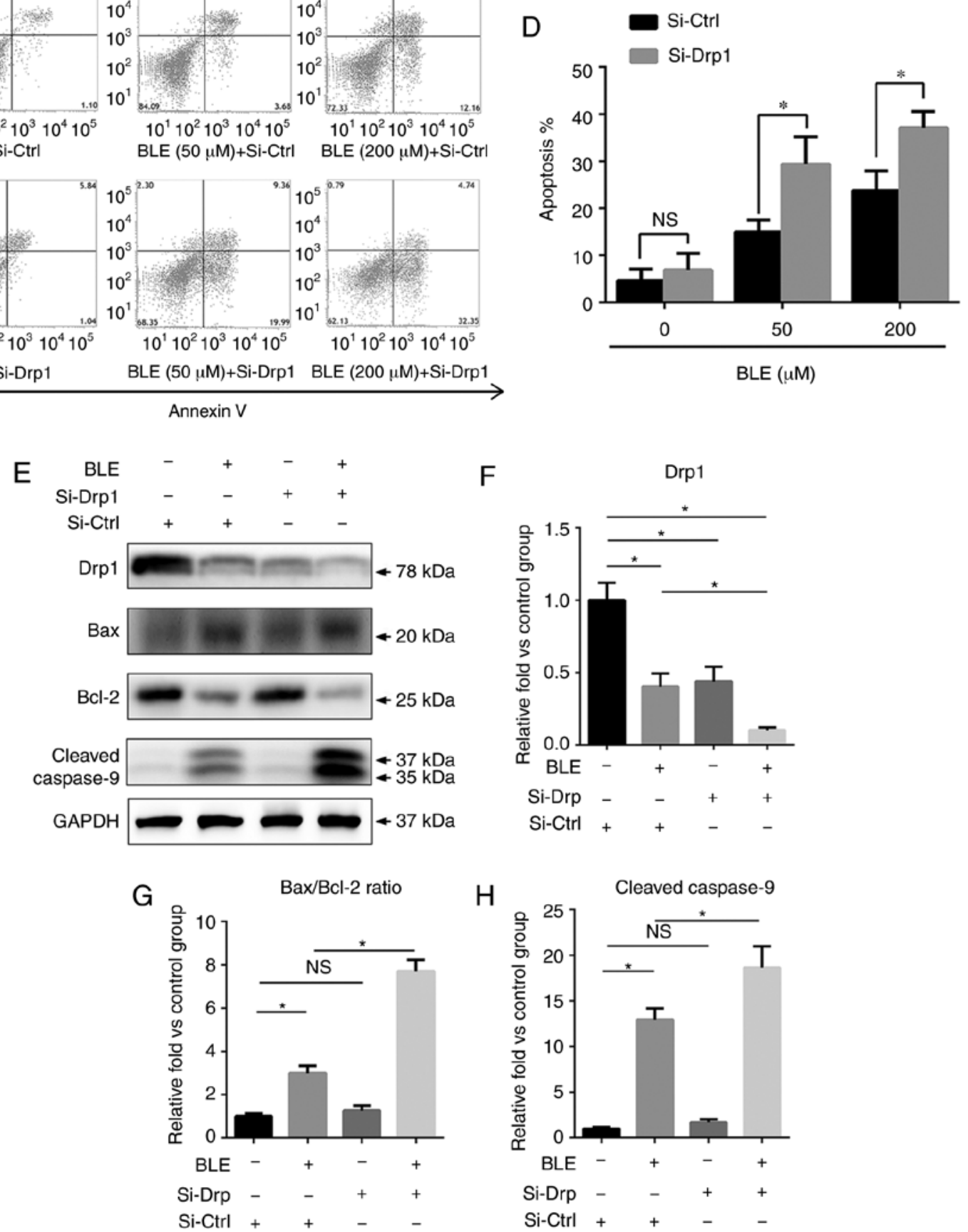

Figure 3. Drp1 knockdown results in increased apoptosis in BLE-A5-treated NPDFs. (A and B) Reverse transcription-PCR and western blot analysis of siRNA-mediated Drp1 knockdown efficiency. (C) Dot plots of Annexin V/PI flow cytometric analysis of Si-scrambled RNA-transfected NPDFs as a control (Si-Ctrl) or knockdown (Si-Drp1) NPDFs, both of which were treated with 50 or $200 \mu \mathrm{M}$ BLE for $48 \mathrm{~h}$. (D) Quantification of the percentage of apoptotic cells. Data were analyzed by Student's t-test. (E) Western blot analysis of proteins associated with mitochondria-mediated apoptosis. NPDFs were first pretreated for $12 \mathrm{~h}$ with Drp1 siRNA or Si-scrambled RNA and then left untreated again or treated with $200 \mu \mathrm{M}$ BLE for $48 \mathrm{~h}$. Quantification of the results in (E) showing the fold-change in (F) Drp1 expression, $(\mathrm{G})$ Bax-Bcl-2 ratio and $(\mathrm{H})$ cleaves caspase-9 normalized to the internal reference, GAPDH. Data were analyzed by one-way ANOVA followed by Sidak's multiple comparisons test. Values are presented as the mean \pm standard deviation. $n=3$. "P<0.05. NS, not significant; NPDF, nasal polyp-derived fibroblast; BLE-A5, bleomycin A5; Drp1, dynamin-related protein 1; Si, small interfering RNA; Ctrl, control. 

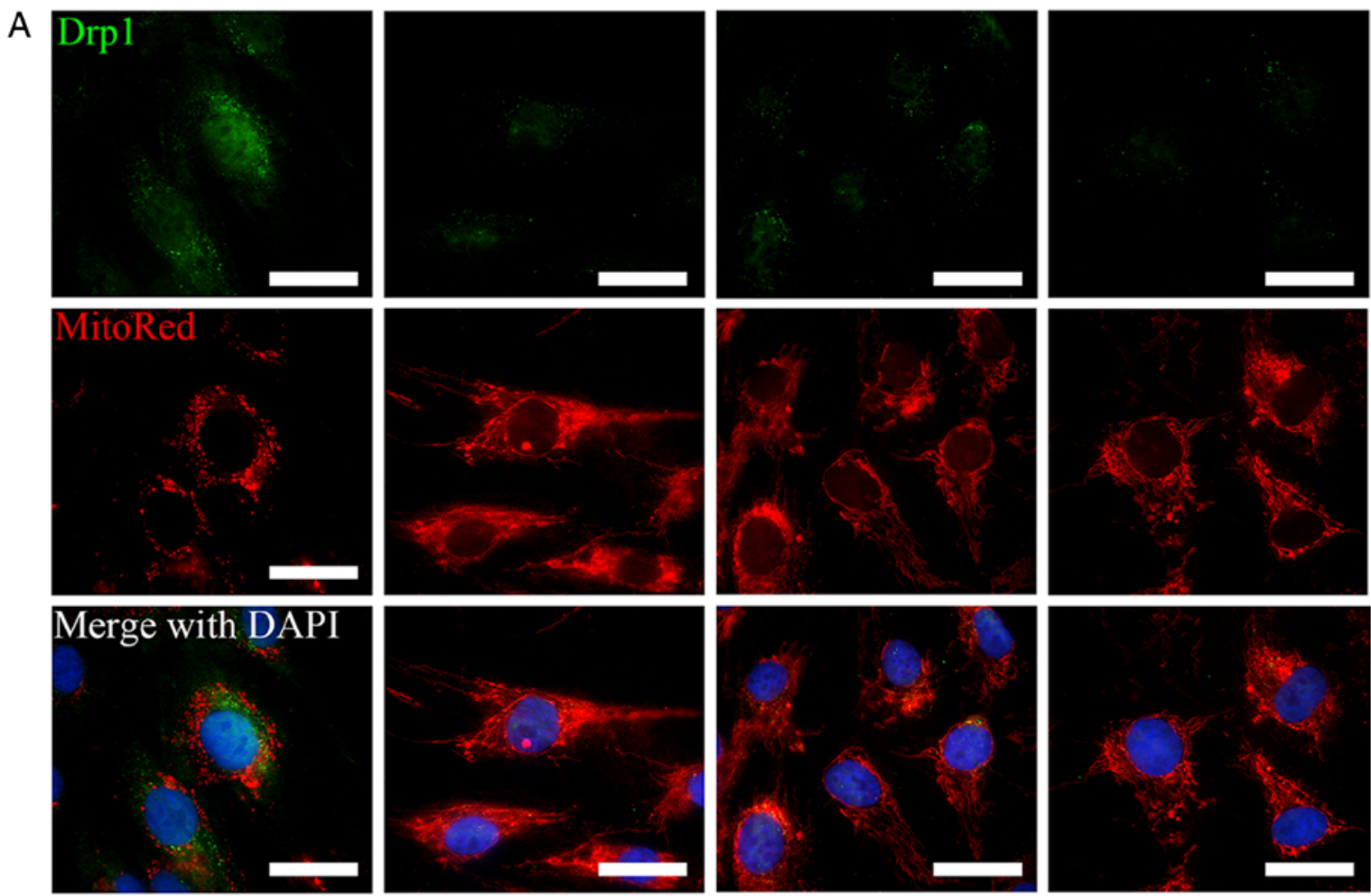

BLE

Si-Drp1

Si-Ctrl
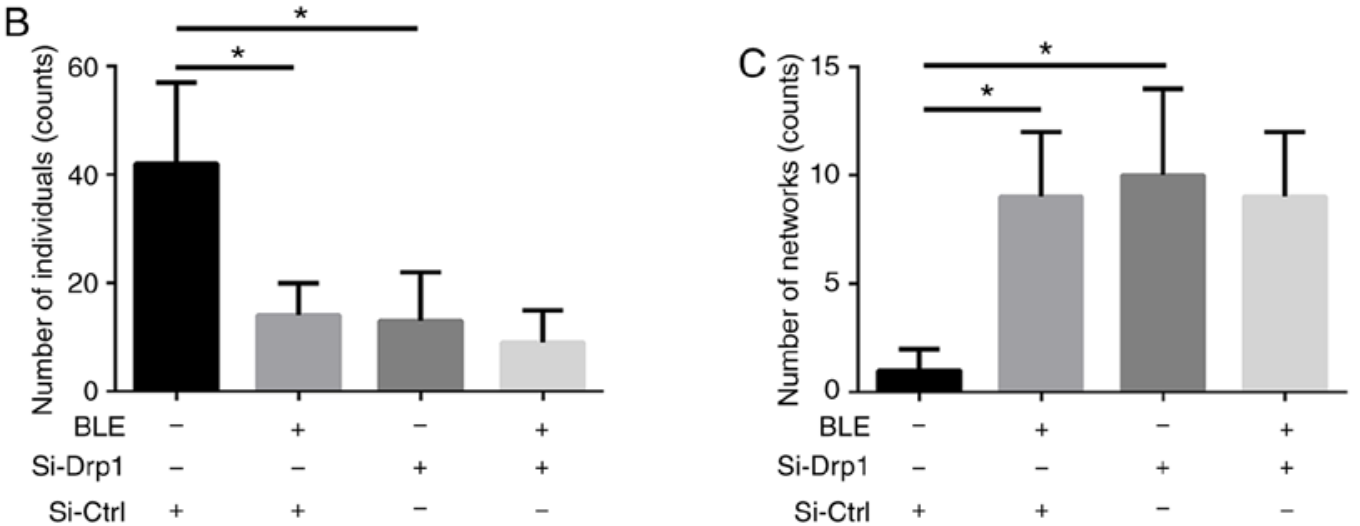

Figure 4. Drp1 knockdown and BLE-A5 treatment alters mitochondrial morphology in NPDFs. (A) Drp1 expression was measured by immunofluorescence using a Drp1 antibody co-stained with MitoRed in Si-scrambled RNA-transfected NPDFs (Si-Ctrl) or Drp1-knockdown (Si-Drp1) NPDFs with or without $200 \mu \mathrm{M}$ BLE-A5 for $48 \mathrm{~h}$. Drp1 is shown in green and mitochondria are shown in red. Scale bar, $20 \mu \mathrm{m}$. Quantification of the number of (B) individual and $(C)$ network mitochondria Values are presented as the mean \pm standard deviation. $n=5$. "P<0.05. Data were analyzed by one-way ANOVA followed by Sidak's multiple comparisons test. NPDF, nasal polyp-derived fibroblast; BLE-A5, bleomycin A5; Drp1, dynamin-related protein 1; Si, small interfering RNA; Ctrl, control.

was similar to Si-Ctrl-transfected cells but decreased below that of the Si-Ctrl group in the presence of 50 or $200 \mu \mathrm{M}$ BLE-A5. Taken together, these results suggested that Drp1 knockdown can aggravate BLE-induced mitochondrial dysfunction in NPDFs.

BLE-A5 treatment inhibits Drpl-mediated mitophagy in NPDFs. The localization of Drp1 and mitophagy-related proteins in mitochondrial and cytosolic fractions of NPDFs were assessed following BLE-A5 treatment (200 $\mu \mathrm{M}$ for $48 \mathrm{~h}$ ) (Fig. 6A). There was a decrease in Drp1 expression in both the mitochondrial and cytosolic fractions of NPDFs treated with BLE-A5, regardless of siRNA transfection (Fig. 6B). Moreover, PINK1 and Parkin, two proteins that are essential for the ubiquitination of dysfunctional mitochondria and subsequent activation of mitophagy, were significantly decreased in the mitochondrial fraction of Si-Drp1-transfected NPDFs compared to Si-Ctrl NPDFs in the presence of BLE-A5 (Fig. 6C and D). These results indicated that BLE can inhibit mitophagy in NPDFs, and Drp1 knockdown can increase BLE-mediated mitophagy blockade in NPDFs.

Autophagy was also assessed by measuring LC3B expression by western blotting and immunofluorescence 
A

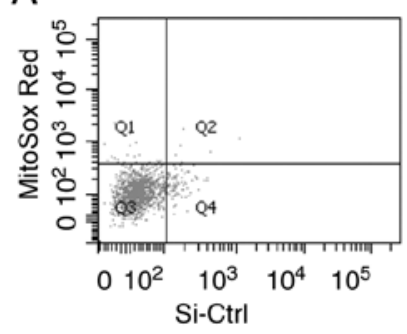

Superoxide analysis

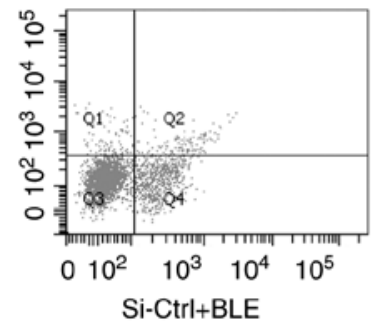

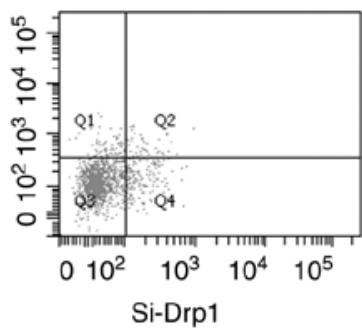

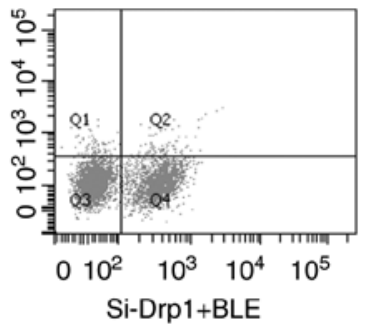

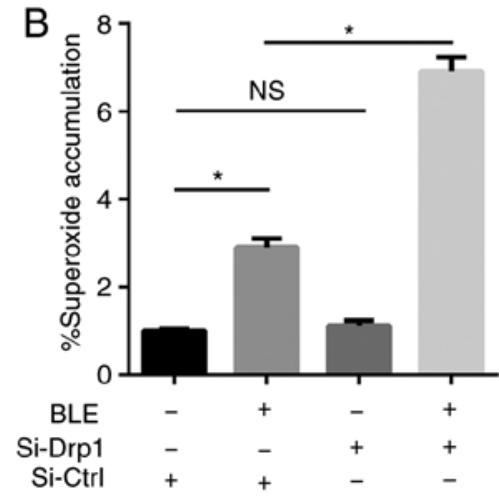
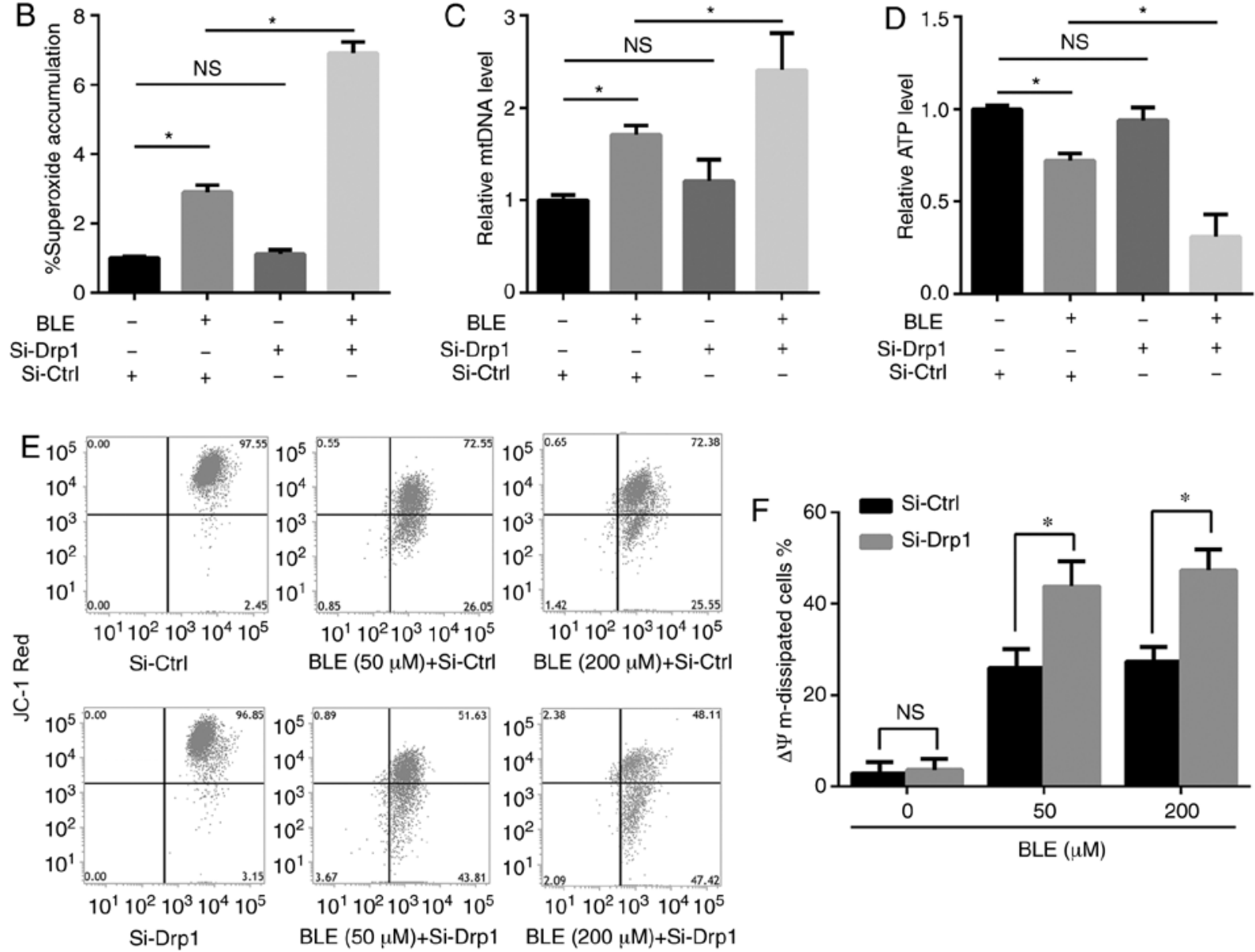

Figure 5. Drp1 knockdown increases BLE-A5-induced deficits in mitochondrial metabolism and mtDNA duplication in NPDFs. Si-Drp1-transfected or Si-scrambled RNA-transfected NPDFs (Si-Ctrl) were treated with or without $200 \mu \mathrm{M}$ BLE-A5 for $48 \mathrm{~h}$. (A) Representative flow cytometry plots of MitoSOX straining. (B) Quantification of superoxide accumulation in different groups. (C) Reverse-transcription quantitative PCR analysis of mtDNA copy number in Drp1-knockdown NPDFs. (D) Intracellular ATP levels were measured by a bioluminescence ATP determination assay. (E) Mitochondrial membrane potential was analyzed using JC-1 reagent. (F) Histogram depicting the percentage of cells with dissipated mitochondrial membrane potential normalized to the control group. Values are presented as the mean \pm standard deviation. $n=5$. "P<0.05. Data were analyzed by one-way ANOVA with Sidak's multiple comparisons test (B-D) or Student's t-test (F). NS, not significant; NPDF, nasal polyp-derived fibroblast; BLE-A5, bleomycin A5; Drp1, dynamin-related protein 1; Si, small interfering RNA; Ctrl, control; mtDNA, mitochondrial DNA.

co-localization with MitoRed (Fig. 6E-H). The results showed that both BLE treatment and Drp1 knockdown decreased LC3B expression, which further confirmed that mitophagy is inhibited by BLE treatment and Drp1 plays a role in BLE-mediated mitophagy inhibition in NPDFs.

BLE-A5 decreases cyclin B1-CDK1 complex-mediated phosphorylation of Drpl in NPDFs. Western blotting showed that BLE-A5 significantly decreased the phosphorylation of Drp1 at serine 616 (Fig. 7A and B), which is required for Drp1 translocation into the mitochondrial membrane. Si-Drp1-transfected NPDFs also showed decreases in
p-Drp1(S616) levels, which decreased further when the cells were treated with BLE-A5 (200 $\mu \mathrm{M}$ for $48 \mathrm{~h})$. Quantification of the $\mathrm{p}$-Drp1/Drp1 ratio confirmed a significant decrease in either siDrp1 or BLE-A5 treated NPDFs.

To confirm the association between the decreased p-Drp1(S616) levels and the changes in mitochondrial morphology, a FITC-conjugated secondary antibody (green fluorescence) was used to examine p-Drp1(S616) localization in NPDFs co-stained with MitoRed. Fluorescence microscopy showed that when NPDFs were treated with BLE-A5, the decrease in green fluorescence was consistent with mitochondrial fusion (indicated by long tubular networks) (Fig. 7C-E). 

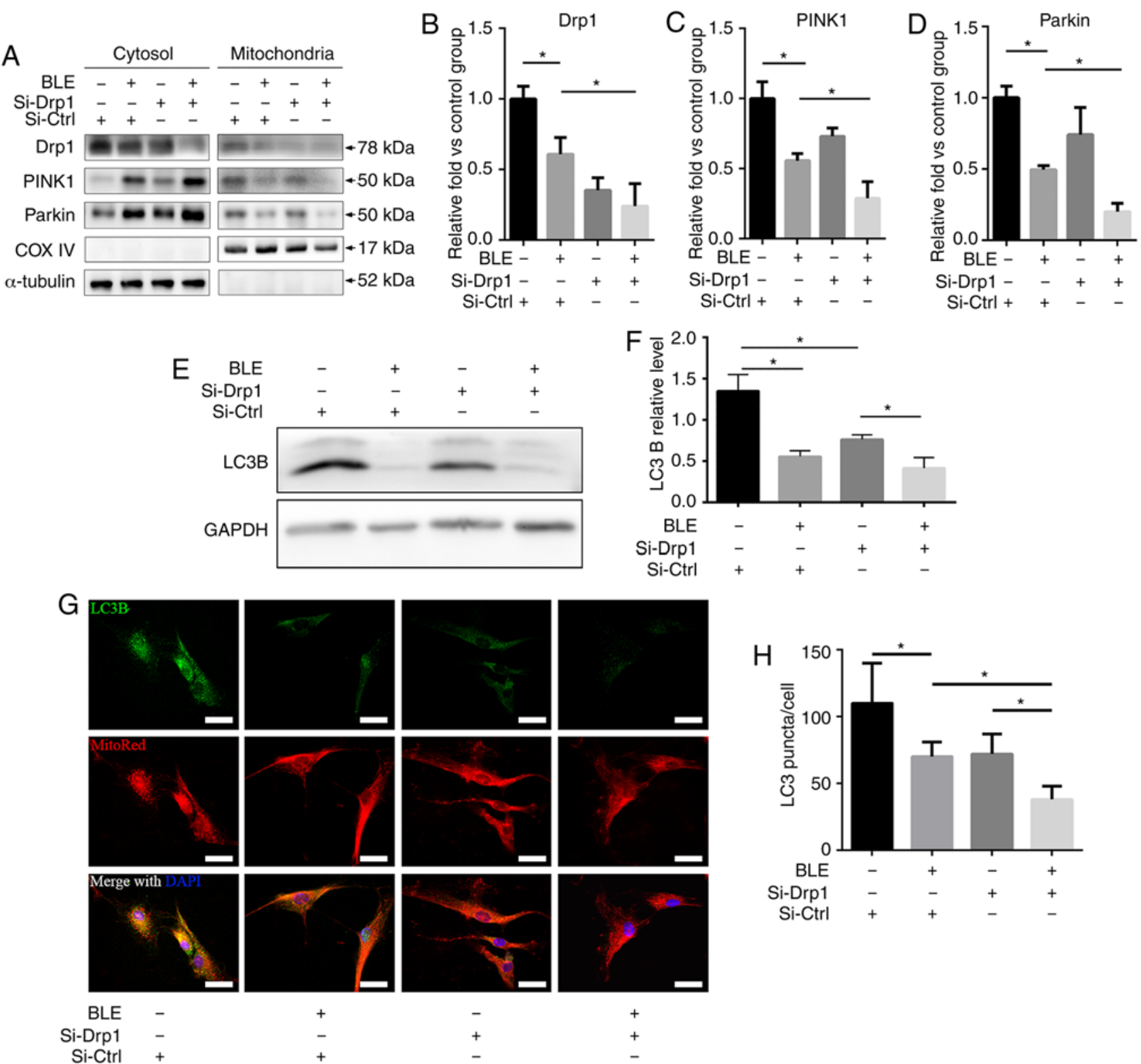

Figure 6. BLE-A5 treatment inhibits mitophagy and Drp1 knockdown increases this inhibitory effect in NPDFs. (A) Western blot analysis of Drp1 and mitophagy-related protein expression (PINK1 and Parkin) in mitochondrial and cytosolic fractions. The protein levels of $\alpha$-tubulin were used to confirm equal loading of cytosolic proteins, and the protein levels of COX IV were used to confirm equal loading of mitochondrial proteins. Quantification of (B) Drp1, (C) PINK1 and (D) Parkin protein expression. (E) Western blot analysis of LC3B expression. The protein levels of GAPDH was used to confirm equal loading. (F) Quantification of LC3B protein expression. (G) Immunofluorescence imaging of LC3B puncta co-localized with MitoRed. (H) Quantification of LC3B-positive puncta. Values are presented as the mean \pm standard deviation. $n=3-6$. "P<0.05. Data were analyzed by one-way ANOVA with Sidak's multiple comparisons test. NPDF, nasal polyp-derived fibroblast; BLE-A5, bleomycin A5; Drp1, dynamin-related protein 1; Si, small interfering RNA; Ctrl, control; PINK1, serine/threonine kinase PINK1; COX IV, cyclooxygenase IV.

The cyclin B1-CDK1 complex is essential for the phosphorylation of Drp1 [p-Drp1(S616)] during mitochondrial fission (42). Co-immunoprecipitation was performed to examine the effects of BLE-A5 treatment on cyclin B1-CDK1 complex formation in NPDFs. It was found that BLE-A5 reduced the levels of cyclin B1-CDK1 complex formation (Fig. 7F), thereby suppressing Drp1 phosphorylation and subsequent mitochondrial translocation. RO-3306 is a well-known CDK1 inhibitor. When treated with RO-3306, NPDFs showed a significant decrease in p-Drp1 expression (Fig. 7G), but no obvious change in total Drp1 levels. Thus, a significant decrease in the $\mathrm{p}$-Drp1/Drp1 ratio was observed in RO-3306-treated cells (Fig. 7H).

\section{Discussion}

As a traditional antitumor drug, the clinical application of BLE-A5 has been limited by severe complications, such as lung fibrosis and scleroderma (43). However, intralesional injection of BLE-A5 into keloids, hypertrophic scars (44) and maxillofacial hemangiomas (45) have proven to be effective and safe treatment alternatives. In addition, BLE-A5 injections have already been used to treat NPs in China, especially in difficult-to-treat, glucocorticoid-insensitive and recurrent cases $(22,23)$. One important stage in the pathology of NPs is irreversible tissue remodeling, which is mainly driven by fibroblasts (NPDFs) that respond to inflammation and produce 

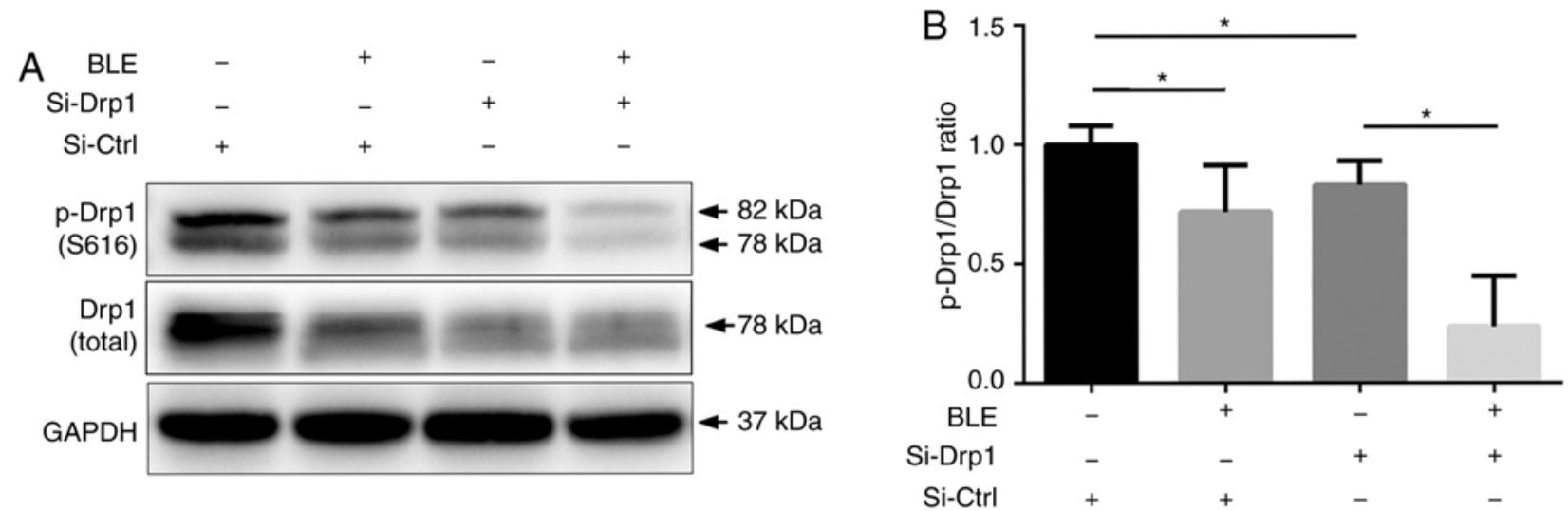

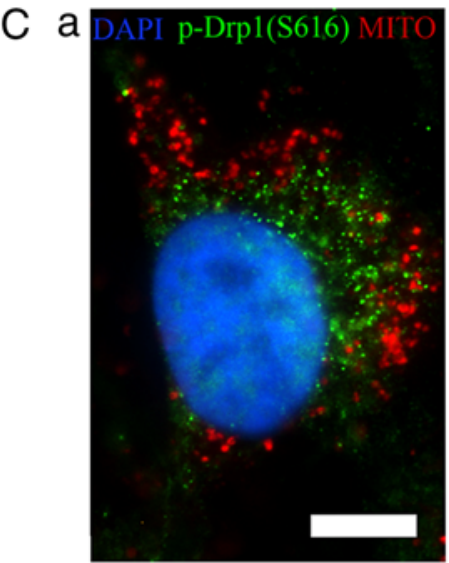

NC

$\mathrm{F}$

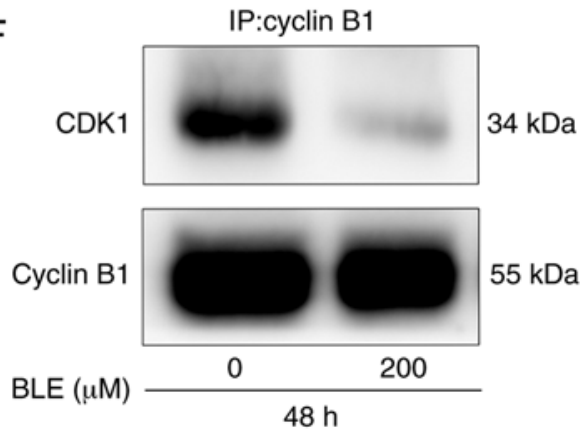

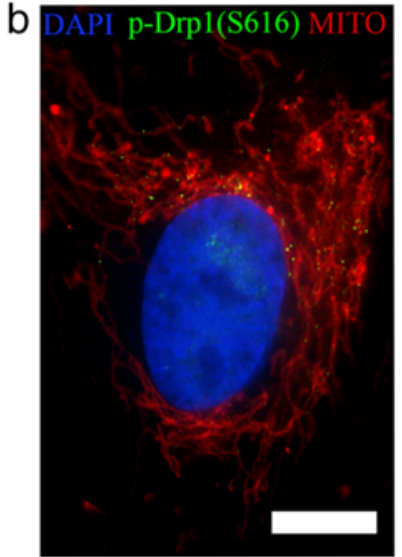

BLE $(200 \mu \mathrm{M})$
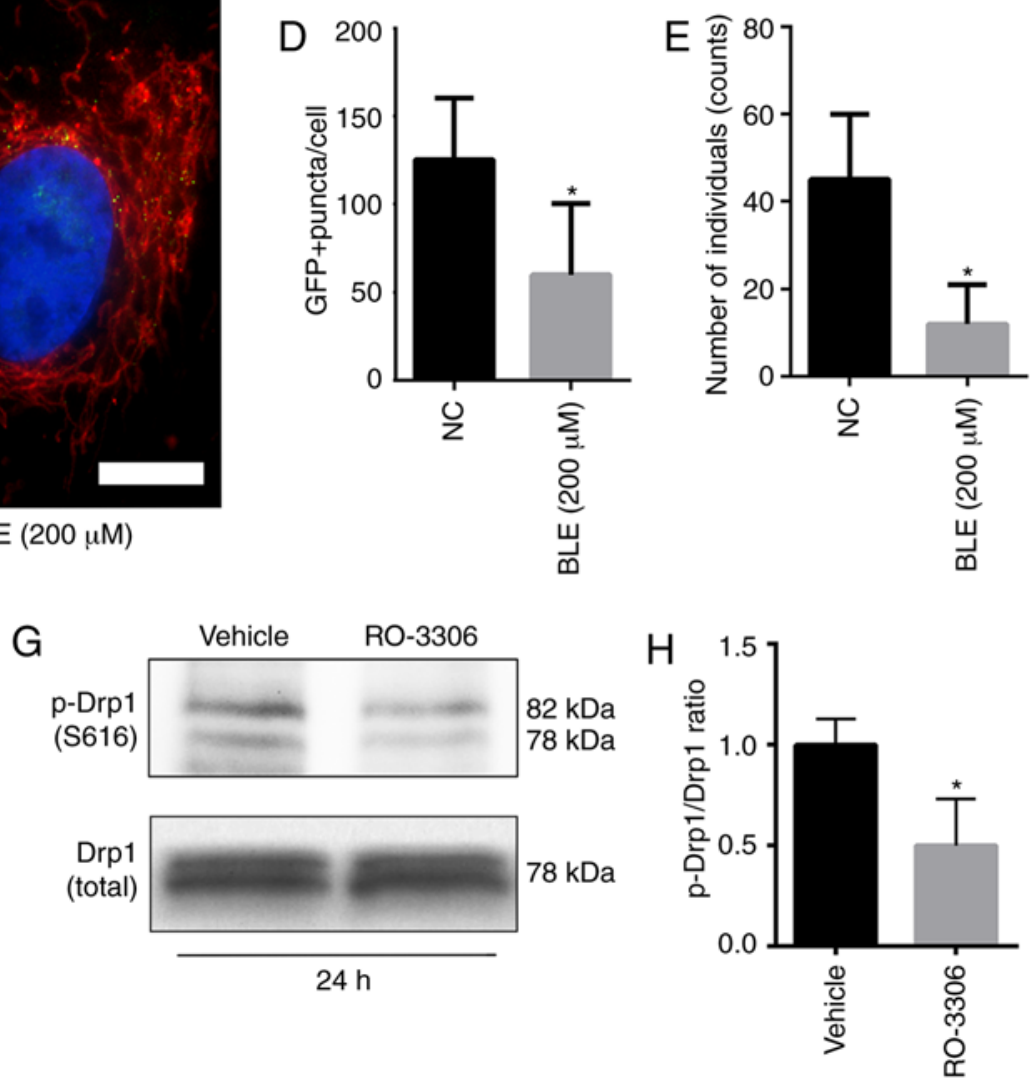

Figure 7. BLE treatment decreases S616 phosphorylation of Drp1 in NPDFs. (A) Cell lysates from NPDFs under different conditions were immunoblotted for p-Drp1 (S616). (B) Quantification of p-Drp1 levels shown in (A). (C) Immunofluorescence analysis of p-Drp1(S616) co-staining with MitoRed. (Ca) Untreated NPDFs. (Cb) NPDFs treated with $200 \mu$ M BLE-A5. DAPI is shown in blue, p-Drp1(S616) is shown in green and mitochondria are shown in red. Scale bar, $5 \mu \mathrm{m}$. Quantification of (D) GFP-positive puncta and (E) the number of individual mitochondria. (F) The effects of BLE treatment on the expression of the cyclin B1-CDK1 complex in NPDFs was determined by co-immunoprecipitation with anti-cyclin-B1, followed by immunoblotting for CDK1 and cyclin B1. (G) Treatment with the CDK1 inhibitor RO-3306 decreased the levels of p-Drp1 in NPDFs as shown by western blotting. (H) Quantification of p-Drp1 levels shown in (G). Values are presented as the mean \pm standard deviation. $n=3$. "P $<0.05$. Data were analyzed by one-way ANOVA with Sidak's multiple comparisons test (B) or Student's t-test (D, E and H). MITO, mitochondria; IP, immunoprecipitation; NPDF, nasal polyp-derived fibroblast; BLE-A5, bleomycin A5; $\mathrm{NC}$, negative control; GFP, green fluorescent protein; p, phosphorylated; Drp1, dynamin-related protein 1; Si, small interfering RNA; Ctrl, control.

extracellular matrix proteins (46). The present study first performed immunofluorescence TUNEL co-localization analysis. The results showed that BLE-A5-induced apoptosis mainly occurred in fibroblasts. This result was consistent with the clinical effects showing that BLE-A5 injection can effectively reduce polyp volume (22). It is well documented that nasal polyps are reconstructed tissues which are initiated by the immune response, including inflammatory cell infiltration and inflammatory factor secretion. However, the present study mainly focused on the pro-apoptotic effects of BLE-A5 on NPDFs. Further studies still need to be performed to assess the effects of BLA-A5 in nasal polyp inflammation response.

The present study revealed that BLE-A5 could decrease the expression of Drp1 and induce mitochondrial pathway-mediated apoptosis in NPDFs in a time- and dose-dependent manner. BLE-A5-treated NPDFs exhibited an early phase 

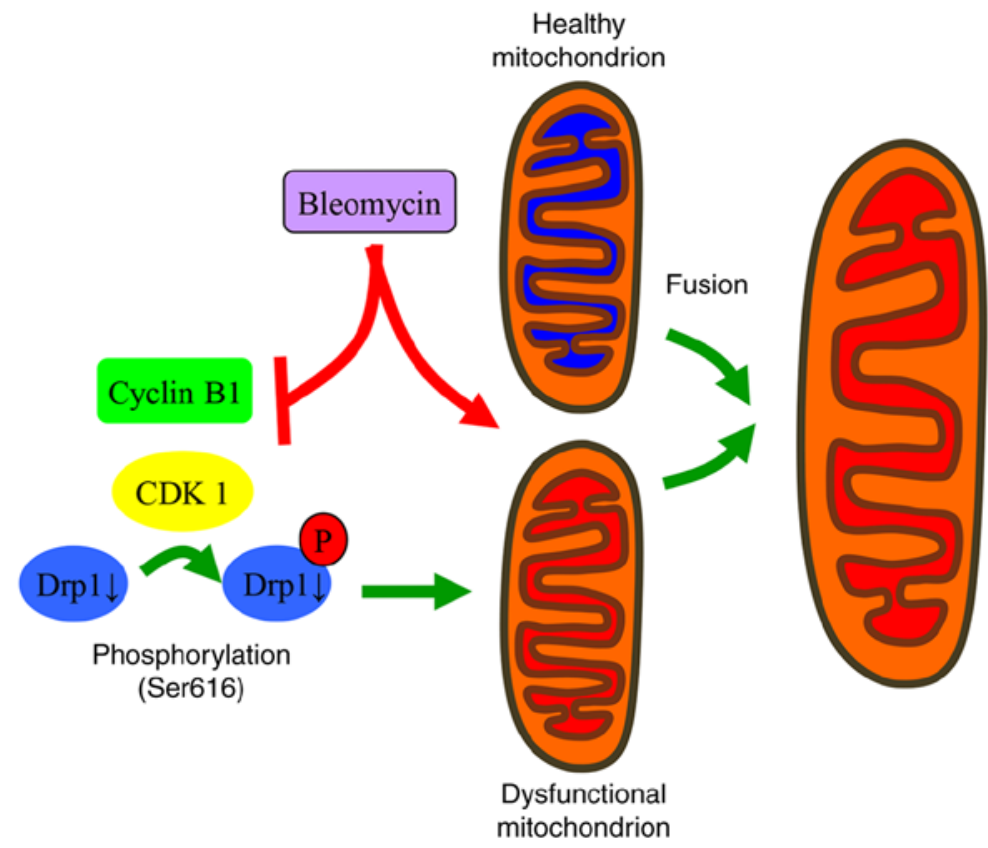

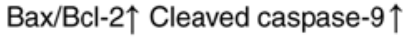
$\mathrm{ROS} \uparrow$

$\mathrm{PINK} 1 \downarrow \mathrm{PARKIN} \downarrow$ $\Delta \Psi \mathrm{m} \downarrow$

Figure 8. Proposed mechanisms by which Drp1 mediates the changes in mitochondrial morphology and function in response to BLE treatment in NPDFs. BLE-A5 treatment can lead to mitochondrial dysfunction and suppress the formation of active cyclin B1-CDK1 complexes. This suppression inhibits the phosphorylation of Drp1 and its translocation to the mitochondrial membrane, thus increasing the fusion of dysfunctional and healthy mitochondria. Subsequently, ROS production increases, mitochondrial membrane potential dissipates, and apoptosis is induced in NPDFs. NPDF, nasal polyp-derived fibroblast; Drp1, dynamin-related protein 1; ROS, reactive oxygen species; P, phosphorylation; PINK1, serine/threonine kinase PINK1; C-Cas9, cleaved caspase-9.

increase in Drp1 expression at the 12-24 h time points, which then sharply decreased after $48 \mathrm{~h}$, and the increase in cleaved caspase-9 levels occurred at 48-72 h. These results match our previous findings showing that $200 \mu \mathrm{M}$ BLE-A5 induced NPDF apoptosis mainly after $48 \mathrm{~h}$ (27). In the early phase (between 12-24 h), BLE-A5-treated NPDFs did not undergo obvious apoptosis; however, cellular ROS were increased (28). Therefore, the increase in Drp1 expression may be due to the ROS stress response. It was reported that ROS generation can effectively increase Drp1 expression (47). However, ROS accumulation in BLE-A5-treated NPDFs only occurs in the early phase (28). After $24 \mathrm{~h}$, ROS levels decreased to normal levels, and so Drp1 decreased accordingly. When the cells were treated for $48 \mathrm{~h}$, the suppressive effects of BLE-A5 on Drp1 expression dominated, and hence a sharp decrease in Drp1 expression was observed. Additionally, Si-Drp1-transfected NPDFs were more sensitive to BLE-induced apoptosis compared with control cells. Theoretically, Drp1 overexpression should be able to abolish the pro-apoptotic effects of BLE-A5 in NPDFs. However, the present study attempted to overexpress Drp1 in NPDFs using viral vectors in preliminary experiments. However, the results showed that Drp1 overexpression in NPDFs cannot increase p-Drp1 accordingly and did not show protective effects against BLE-A5 administration. p-Drp1 is the activated form of Drp-1 that performs mitochondrial pro-fission functions (48). Because no commercial specific Drp-1 activator is available, the present study was unable to perform rescue experiments. Moreover, it was found that BLE-A5 treatment could change the morphology of mitochondria in NPDFs, transforming them from short tubules or round fragments into long tubular networks. Furthermore, Si-Drp1-transfected NPDFs showed similar mitochondrial morphology as that of Si-Ctrl-transfected cells treated with BLE-A5. However, simultaneous Drp1 silencing and BLE-A5 treatment showed no further changes in the mitochondrial structure compared to Si-Drp1 or BLE-A5-treated NPDFs. This phenomenon may due to either Si-Drp1 or BLE-A5 treatment under these conditions have hit the limit of mitochondrial fusion; hence, the overall structure showed no further change by co-administration. It was also found that Drp1 knockdown in NPDFs increased mitochondrial dysfunction when the cells were exposed to BLE-A5 and that the PINK1-Parkin-mediated mitophagy pathway was inhibited more severely in Si-Drp1-transfected NPDFs compared with Si-Ctrl-transfected cells when exposed to BLE. These findings were further supported by showing that BLE-A5 could suppress the formation of the cyclin B1-CDK1 complex, thereby decreasing Drp1 phosphorylation.

Bcl-2 and Bax are two essential proteins that regulate mitochondria-mediated apoptosis. Pro-apoptotic Bax can form a complex with the anti-apoptotic $\mathrm{Bcl}-2$; thus, the $\mathrm{Bax} / \mathrm{Bcl}-2$ ratio determines whether a cell will undergo apoptosis (49). Caspase-9 is the protease that initiates the mitochondria-mediated apoptotic pathway and is activated by multiprotein activation platforms (50). The present study showed that BLE-A5 treatment altered the $\mathrm{Bax} / \mathrm{Bcl}-2$ ratio and activated caspase-9, thus initiating mitochondria-mediated apoptosis. Furthermore, the Bax/Bcl-2 ratio and cleaved caspase-9 expression was higher in Si-Drp1-transfected NPDFs than in Si-Ctrl-transfected cells exposed to a clinical dose of BLE-A5 $(200 \mu \mathrm{M})$, indicating that Drp1 could reduce the activation of mitochondria-mediated apoptosis in BLE-treated NPDFs.

Mitochondria are double membrane-bound, subcellular organelles that regulate a host of metabolic functions and are 
closely associated with the intrinsic apoptosis pathway (51). When a cell undergoes internal or external stress, its mitochondria take quality-control steps to eliminate damaged proteins, lipids and DNA by isolating damaged factors and transferring them to the lysosome for degradation (52). This mitochondrial fission process is mediated by large GTPases in the dynamin family (53). Among these GTPases, Drp1 is known to play a critical role in regulating mitochondrial fission in cells under stress (54). The present study found that a low dose of BLE-A5 could enhance Drp1 expression NPDFs, but a clinical dose could decrease Drp1 expression and change the morphology of mitochondria from fragments into networks. To examine whether such changes play a protective or pro-apoptotic role during BLE exposure, Drp1 expression in NPDFs was knocked down via siRNA transfection. The results showed that Si-Drp1-transfected NPDFs were more sensitive to BLE-A5-induced apoptosis than Si-Ctrl-transfected cells, indicating that the BLE-induced decrease in Drp1 expression may shut down the quality-control mechanisms of mitochondria in NPDFs, rendering these cells unable to eliminate cytotoxic factors.

ATP, the 'energy currency' of the cell, is essential for cellular metabolism and is primarily produced by mitochondria. However, mitochondria also continually produce harmful ROS as a byproduct of electron transport during oxidative phosphorylation (55). Mitochondria contain their own self-replicating genomes (mtDNA) that encode essential protein components of the electron transport chain (56). The mitochondrial membrane potential $(\Delta \psi \mathrm{m})$ can reflect the functional status of mitochondria (57). ROS-induced stress can cause the accumulation of mutated mtDNA in human cells $(58,59)$. A study showed that BLE could induce lung epithelial cell apoptosis via activation of the ROS/Akt signaling pathway and damage mtDNA $(60,61)$. Consistent with these previous studies, the present results showed that BLE exposure in NPDFs enhanced ROS and mtDNA levels, inhibited ATP generation and caused the mitochondrial membrane potential to depolarize. Moreover, these indications of BLE-induced mitochondrial dysfunction were more significant in Si-Drpl-transfected NPDFs than in control cells, indicating that the self-protective mechanism of Drp1-mediated mitochondrial fission was further suppressed by BLE-A5 treatment.

When stress is not sufficient to induce apoptosis, an important mechanism that protects cells from the harmful accumulation of mtDNA caused by excessive ROS is the activation of mitophagy (62). Studies of PINK1-Parkin-mediated mitophagy have yielded insight into a molecular quality control mechanism via the elimination of damaged mitochondria (63-65). When a mitochondrion becomes damaged, PINK1 accumulates in the mitochondrial outer membrane, which then recruits Parkin from the cytosol (66). Parkin conjugates ubiquitin to a variety of proteins on the outer mitochondrial membrane to generate an autophagosome, which is later degraded by lysosomal hydrolases (67). The present study found that BLE treatment could suppress such protective mechanisms in NPDFs by blocking the mitochondrial accumulation of PINK1-Parkin. Moreover, this suppression was enhanced by Drpl knockdown, indicating the necessity of Drp1 in the activation of PINK1-Parkin-mediated mitophagy. LC3B was measured by western blotting and immunofluorescence, and the findings also supported this theory.

It was reported that Drp1 activation depends on the cyclin B1-CDK1 complex-mediated phosphorylation of Drp1 at S616 and subsequent localization on the outer mitochondrial membrane (68). The present results showed that BLE treatment reduced the phosphorylation of Drp1 at S616 and its localization in mitochondria. Si-Drp1-transfected NPDFs expressed lower levels of p-Drp1(S616), indicating that knockdown of total Drp1 expression could also decrease p-Drp1(S616) levels and thereby suppress its function in mitochondrial fission. The co-immunoprecipitation results showed that BLE suppressed the formation of the cyclin B1-CDK1 complex and thus decreased the phosphorylation of Drp1. CDK1 inhibition by RO-3306 decreased p-Drp1(S616) expression in NPDFs, which also provided direct evidence that CDK1 is required for the activation of Drp1.

In summary, the present findings suggested that BLE inhibits the interaction of cyclin B1 with CDK1 and decreases the expression of total Drp1 in NPDFs, thus decreasing the levels of p-Drp1 (S616). This decreased expression blocks the protective mechanisms of mitochondrial fission and PINK1-Parkin-mediated mitophagy to eliminate dysfunctional mitochondria. In this manner, BLE can effectively induce NPDF apoptosis (Fig. 8). Further studies are still needed to evaluate the safety and effectiveness of such treatments.

\section{Acknowledgements}

Not applicable.

\section{Funding}

This study was funded by the National Natural Science Foundation of China (grant no. 81500773) and the Natural Science Foundation of Guangdong Province of China (grant no. 2015A030310125).

\section{Availability of data and materials}

The datasets used and/or analyzed during the current study are available from the corresponding author on reasonable request.

\section{Authors' contributions}

FW, YM, JW and HO performed the experiments. FW analyzed the data and wrote the manuscript. HD, YZ, PT and HZ conceptualized the study design, and contributed to data analysis and experimental materials. All authors read and approved the final manuscript.

\section{Ethics approval and consent to participate}

The study was approved by the Ethics Committee of Sun Yat-sen Memorial Hospital (approval no. SYSU81500773; Guangzhou, China).

\section{Patient consent for publication}

Not applicable. 


\section{Competing interests}

The authors declare that they have no competing interests.

\section{References}

1. Hulse KE, Stevens WW, Tan BK and Schleimer RP: Pathogenesis of nasal polyposis. Clin Exp Allergy 45: 328-346, 2015.

2. Schleimer RP: Immunopathogenesis of chronic rhinosinusitis and nasal polyposis. Annu Rev Pathol 12: 331-357, 2017.

3. Klossek JM, Neukirch F, Pribil C, Jankowski R, Serrano E, Chanal I and El Hasnaoui A: Prevalence of nasal polyposis in France: A cross-sectional, case-control study. Allergy 60 233-237, 2005

4. Hedman J, Kaprio J, Poussa T and Nieminen MM: Prevalence of asthma, aspirin intolerance, nasal polyposis and chronic obstructive pulmonary disease in a population-based study. Int $\mathbf{J}$ Epidemiol 28: 717-722, 1999.

5. Settipane GA and Chafee FH: Nasal polyps in asthma and rhinitis. A review of 6,037 patients. J Allergy Clin Immunol 59: 17-21, 1977.

6. Ahn JC, Kim JW, Lee CH and Rhee CS: Prevalence and risk factors of chronic rhinosinusitus, allergic rhinitis, and nasal septal deviation: Results of the Korean national health and nutrition survey 2008-2012. AMA Otolaryngol Head Neck Surg 142: 162-167, 2016.

7. Stevens WW, Schleimer RP and Kern RC: Chronic rhinosinusitis with nasal polyps. J Allergy Clin Immunol Pract 4: 565-572, 2016.

8. Kato A: Immunopathology of chronic rhinosinusitis. Allergol Int 64: 121-130, 2015

9. Dobzanski A, Khalil SM and Lane AP: Nasal polyp fibroblasts modulate epithelial characteristics via Wnt signaling. Int Forum Allergy Rhinol 8: 1412-1420, 2018.

10. Veloso-Teles R, Cerejeira R, Roque-Farinha R and Buchwald CV: Systemic immune profile in patients with CRSwNP. Ear Nose Throat J: Dec 4, 2019 (Epub Ahead of Print).

11. Zhang Y, Gevaert E, Lou H, Wang X, Zhang L, Bachert C and Zhang N: Chronic rhinosinusitis in Asia. J Allergy Clin Immunol 140: 1230-1239, 2017.

12. Meng J, Zhou P, Liu Y, Liu F, Yi X, Liu S, Holtappels G, Bachert $\mathrm{C}$ and Zhang $\mathrm{N}$ : The development of nasal polyp disease involves early nasal mucosal inflammation and remodelling. PLoS One 8: e82373, 2013.

13. Cho JS, Kang JH, Um JY, Han IH, Park IH and Lee HM: Lipopolysaccharide induces pro-inflammatory cytokines and MMP production via TLR4 in nasal polyp-derived fibroblast and organ culture. PLoS One 9: e90683, 2014.

14. Cho JS, Han IH, Lee HR and Lee HM: Prostaglandin E2 induces IL- 6 and IL- 8 production by the EP receptors/Akt/NF- $\kappa$ B pathways in nasal polyp-derived fibroblasts. Allergy Asthma Immunol Res 6: 449-457, 2014.

15. Park IH, Park SJ, Cho JS, Moon YM, Kim TH, Lee SH and Lee HM: Role of reactive oxygen species in transforming growth factor beta1-induced alpha smooth-muscle actin and collagen production in nasal polyp-derived fibroblasts. Int Arch Allergy Immunol 159: 278-286, 2012

16. Thomas M, Yawn BP, Price D, Lund V, Mullol J and Fokkens W; European Position Paper on Rhinosinusitis and Nasal Polyps Group: EPOS primary care guidelines: European position paper on the primary care diagnosis and management of rhinosinusitis and nasal polyps 2007-a summary. Prim Care Respir J 17: 79-89, 2008.

17. Fernandez-Bertolin L, Mullol J, Fuentes-Prado M, Roca-Ferrer J, Alobid I, Picado C and Pujols L: Effect of lipopolysaccharide on glucocorticoid receptor function in control nasal mucosa fibroblasts and in fibroblasts from patients with chronic rhinosinusitis with nasal polyps and asthma. PLoS One 10: e125443, 2015.

18. Embid C, Fernández-Bertolin L, Pujols L, Alobid I, Mullol J and Picado C: Nuclear translocation of the glucocorticoid receptor in fibroblasts of asthmatic patients with nasal polyposis insensitive to glucocorticoid treatment. Arch Bronconeumol 47: 115-121, 2011 (In English, Spanish).

19. Pujols L, Fuentes-Prado M, Fernández-Bertolin L, Alobid I, Roca-Ferrer J, Mullol J and Picado C: Lower sensitivity of nasal polyp fibroblasts to glucocorticoid anti-proliferative effects. Respir Med 105: 218-225, 2011.

20. Bhattacharyya N: Influence of polyps on outcomes after endoscopic sinus surgery. Laryngoscope 117: 1834-1838, 2007.
21. Wynn R and Har-El G: Recurrence rates after endoscopic sinus surgery for massive sinus polyposis. Laryngoscope 114: 811-813, 2004.

22. Zhang X, Zou J, Sun B, Liang J, Zhao L and Li B: Study on the treatment of nasal polyposis with bleomycin A5 local injection. Lin Chuang Er Bi Yan Hou Ke Za Zhi 20: 51-53, 2006 (In Chinese).

23. Zhang X, Zou J, Li B, Ren X and Shi J: Eosinophil apoptosis in nasal polyposis tissue after bleomycin A5 local injection. Lin Chuang Er Bi Yan Hou Ke Za Zhi 18: 279-281, 2004 (In Chinese).

24. Tian P, Wu F, Wang J, Ou H, Liu X, Chen Q, Dang H, Zheng Y, Zhang $\mathrm{X}$ and Zou H: Intralesional bleomycin A5 injection for the treatment of nasal polyps through inducing apoptosis. Acta Otolaryngol 138: 475-482, 2018.

25. Patel PR, Hegde ML, Theruvathu J, Mitra SA, Boldogh I and Sowers L: Norepinephrine reduces reactive oxygen species (ROS) and DNA damage in ovarian surface epithelial cells. J Bioanal Biomed 7: 75-80, 2015.

26. Kucuksayan E, Cort A, Timur M, Ozdemir E, Yucel SG and Ozben T: N-acetyl-L-cysteine inhibits bleomycin induced apoptosis in malignant testicular germ cell tumors. J Cell Biochem 114: 1685-1694, 2013.

27. Wu F, Tian P, Ma Y, Wang J, Ou H and Zou H: Induction of apoptosis in nasal polyp-derived fibroblasts by bleomycin A5 in vitro. Mol Med Rep 17: 5384-5389, 2018

28. Wu F, Tian P, Ma Y, Wang J, Ou H and Zou H: Reactive oxygen species are necessary for bleomycin A5-induced apoptosis and extracellular matrix elimination of nasal polyp-derived fibroblasts. Ann Otol Rhinol Laryngol 128: 135-144, 2019.

29. Nasrallah CM and Horvath TL: Mitochondrial dynamics in the central regulation of metabolism. Nat Rev Endocrinol 10: 650-658, 2014.

30. Marsboom G, Toth PT, Ryan JJ, Hong Z, Wu X, Fang YH, Thenappan T,Piao L,Zhang HJ,Pogoriler J, et al: Dynamin-related protein 1-mediated mitochondrial mitotic fission permits hyperproliferation of vascular smooth muscle cells and offers a novel therapeutic target in pulmonary hypertension. Circ Res 110: 1484-1497, 2012.

31. Chang CR and Blackstone C: Cyclic AMP-dependent protein kinase phosphorylation of Drp1 regulates its GTPase activity and mitochondrial morphology. J Biol Chem 282: 21583-21587, 2007.

32. Cribbs JT and Strack S: Reversible phosphorylation of Drp1 by cyclic AMP-dependent protein kinase and calcineurin regulates mitochondrial fission and cell death. Embo Rep 8: 939-944, 2007.

33. Song M, Gong G, Burelle Y, Gustafsson ÅB, Kitsis RN, Matkovich SJ and Dorn GW II: Interdependence of parkinmediated mitophagy and mitochondrial fission in adult mouse hearts. Circ Res 117: 346-351, 2015.

34. Zuo W, Zhang S, Xia CY, Guo XF, He WB and Chen NH: Mitochondria autophagy is induced after hypoxic/ischemic stress in a Drp1 dependent manner: The role of inhibition of Drp1 in ischemic brain damage. Neuropharmacology 86: 103-115, 2014.

35. Kageyama Y, Hoshijima M, Seo K, Bedja D, Sysa-Shah P, Andrabi SA, Chen W, Höke A, Dawson VL, Dawson TM, et al: Parkin-independent mitophagy requires Drp1 and maintains the integrity of mammalian heart and brain. EMBO J 33: 2798-2813, 2014.

36. Suen DF, Norris KL and Youle RJ: Mitochondrial dynamics and apoptosis. Genes Dev 22: 1577-1590, 2008.

37. Sosulski ML, Gongora R, Danchuk S, Dong C, Luo F and Sanchez CG: Deregulation of selective autophagy during aging and pulmonary fibrosis: The role of TGF $\beta 1$. Aging Cell 14: 774-783, 2015.

38. Eiyama A and Okamoto K: PINK1/Parkin-mediated mitophagy in mammalian cells. Curr Opin Cell Biol 33: 95-101, 2015.

39. Jiang H, Zhang C, Tang Y, Zhao J, Wang T, Liu H and Sun X: The regulator of calcineurin 1 increases adenine nucleotide translocator 1 and leads to mitochondrial dysfunctions. J Neurochem 140: 307-319, 2017.

40. Zhang YC, Zuo WQ, Rong QF, Teng GL and Zhang YM: Glucocorticoid receptor expression on acute lung injury induced by endotoxin in rats. World J Emerg Med 1: 65-69, 2010.

41. Livak KJ and Schmittgen TD: Analysis of relative gene expression data using real-time quantitative PCR and the 2(-Delta Delta C(T)) method. Methods 25: 402-408, 2001

42. Yamano $\mathrm{K}$ and Youle RJ: Coupling mitochondrial and cell division. Nat Cell Biol 13: 1026-1027, 2011.

43. Martin WG, Ristow KM, Habermann TM, Colgan JP, Witzig TE and Ansell SM: Bleomycin pulmonary toxicity has a negative impact on the outcome of patients with Hodgkin's lymphoma. $\mathrm{J}$ Clin Oncol 23: 7614-7620, 2005. 
44. Manca G, Pandolfi P, Gregorelli C, Cadossi M and de Terlizzi F: Treatment of keloids and hypertrophic scars with bleomycin and electroporation. Plast Reconstr Surg 132: e621-e630, 2013.

45. Luo QF and Zhao FY: The effects of Bleomycin A5 on infantile maxillofacial haemangioma. Head Face Med 7: 11, 2011.

46. Carroll WW, O'Connell BP, Schlosser RJ, Gudis DA, Karnezis TT, Lawrence LA, Soler ZM and Mulligan JK: Fibroblast levels are increased in chronic rhinosinusitis with nasal polyps and are associated with worse subjective disease severity. Int Forum Allergy Rhinol 6: 162-168, 2016.

47. Hu J, Zhang Y, Jiang X, Zhang H, Gao Z, Li Y, Fu R, Li L, Li J, Cui $\mathrm{H}$ and Gao N: ROS-mediated activation and mitochondrial translocation of CaMKII contributes to Drp1-dependent mitochondrial fission and apoptosis in triple-negative breast cancer cells by isorhamnetin and chloroquine. J Exp Clin Cancer Res 38: 225, 2019

48. Ausman J, Abbade J, Ermini L, Farrell A, Tagliaferro A, Post M and Caniggia I: Ceramide-induced BOK promotes mitochondrial fission in preeclampsia. Cell Death Dis 9: 298, 2018.

49. Sharifi S, Barar J, Hejazi MS and Samadi N: Roles of the Bcl-2/Bax ratio, caspase- 8 and 9 in resistance of breast cancer cells to paclitaxel. Asian Pac J Cancer Prev 15: 8617-8622, 2014.

50. Kim B, Srivastava SK and Kim SH: Caspase-9 as a therapeutic target for treating cancer. Expert Opin Ther Targets 19: 113-127, 2015.

51. Youle RJ and van der Bliek AM: Mitochondrial fission, fusion, and stress. Science 337: 1062-1065, 2012.

52. Hoppins S, Lackner L and Nunnari J: The machines that divide and fuse mitochondria. Annu Rev Biochem 76: 751-780, 2007

53. Tilokani L, Nagashima S, Paupe V and Prudent J: Mitochondrial dynamics: Overview of molecular mechanisms. Essays Biochem 62: 341-360, 2018

54. Westermann B: Mitochondrial fusion and fission in cell life and death. Nat Rev Mol Cell Biol 11: 872-884, 2010.

55. Balaban RS, Nemoto S and Finkel T: Mitochondria, oxidants, and aging. Cell 120: 483-495, 2005.

56. Dorn GN II, Vega RB and Kelly DP: Mitochondrial biogenesis and dynamics in the developing and diseased heart. Genes Dev 29: 1981-1991, 2015

57. Zhang BB, Wang DG, Guo FF and Xuan C: Mitochondrial membrane potential and reactive oxygen species in cancer stem cells. Fam Cancer 14: 19-23, 2015.

58. Eaton JS, Lin ZP, Sartorelli AC, Bonawitz ND and Shadel GS: Ataxia-telangiectasia mutated kinase regulates ribonucleotide reductase and mitochondrial homeostasis. J Clin Invest 117: 2723-2734, 2007.
59. Lee HC, Yin PH, Lu CY, Chi CW and Wei YH: Increase of mitochondria and mitochondrial DNA in response to oxidative stress in human cells. Biochem J 348: 425-432, 2000.

60. Kim SJ, Cheresh P, Jablonski RP, Morales-Nebreda L, Cheng Y, Hogan E, Yeldandi A, Chi M, Piseaux R, Ridge K, et al: Mitochondrial catalase overexpressed transgenic mice are protected against lung fibrosis in part via preventing alveolar epithelial cell mitochondrial DNA damage. Free Radic Biol Med 101: 482-490, 2016.

61. Yang L, Lin Z, Wang Y, Li C, Xu W, Li Q, Yao W, Song Z and Liu G: Nickle(II) ions exacerbate bleomycin-induced pulmonary inflammation and fibrosis by activating the ROS/Akt signaling pathway. Environ Sci Pollut Res Int 25: 4406-4418, 2018.

62. Kowald A and Kirkwood TB: The evolution and role of mitochondrial fusion and fission in aging and disease. Commun Integr Biol 4: 627-629, 2011.

63. Clark IE, Dodson MW, Jiang C, Cao JH, Huh JR, Seol JH, Yoo SJ, Hay BA and Guo M: Drosophila pink1 is required for mitochondrial function and interacts genetically with parkin. Nature 441: 1162-1166, 2006.

64. Park J, Lee SB, Lee S, Kim Y, Song S, Kim S, Bae E, Kim J, Shong M, Kim JM and Chung J: Mitochondrial dysfunction in Drosophila PINK1 mutants is complemented by parkin. Nature 441: 1157-1161, 2006

65. Greene JC, Whitworth AJ, Kuo I, Andrews LA, Feany MB and Pallanck LJ: Mitochondrial pathology and apoptotic muscle degeneration in Drosophila parkin mutants. Proc Natl Acad Sci USA 100: 4078-4083, 2003.

66. Barodia SK, Creed RB and Goldberg MS: Parkin and PINK1 functions in oxidative stress and neurodegeneration. Brain Res Bull 133: 51-59, 2017.

67. Lazarou M, Sliter DA, Kane LA, Sarraf SA, Wang C, Burman JL, Sideris DP, Fogel AI and Youle RJ: The ubiquitin kinase PINK1 recruits autophagy receptors to induce mitophagy. Nature 524: 309-314, 2015.

68. Taguchi N, Ishihara N, Jofuku A, Oka T and Mihara K: Mitotic phosphorylation of dynamin-related GTPase Drp1 participates in mitochondrial fission. J Biol Chem 282: 11521-11529, 2007.

This work is licensed under a Creative Commons Attribution-NonCommercial-NoDerivatives 4.0 International (CC BY-NC-ND 4.0) License. 